\title{
tAF-MUSICAL: Autofluorescence Super-resolution Microscopy for Molecular Histopathology of Matrix Proteins in Fibrotic Diseases
}

\section{Biswajoy Ghosh}

Indian Institute of Technology Kharagpur

Jyotirmoy Chatterjee

Indian Institute of Technology Khaagpur

\section{Ranjan Paul}

Guru Nanak Institute of Dental Science

\section{Pooja Lahiri}

Indian Institute of Technology Kharagpur

\section{Mousumi Pal}

Guru Nanak Institute of Dental Science

\section{Pabitra Mitra}

Indian Institute of Technology Kharagpur

\section{Balpreet Singh Ahluwalia}

The Arctic University of Norway https://orcid.org/0000-0001-7841-6952

Krishna Agarwal (D krishna.agarwal@uit.no)

The Arctic University of Norway

\section{Article}

Keywords: tissue autofluorescence, MUSICAL, Fibrosis

Posted Date: December 2nd, 2020

DOI: https://doi.org/10.21203/rs.3.rs-101219/v1

License: (c) (1) This work is licensed under a Creative Commons Attribution 4.0 International License.

Read Full License 


\title{
tAF-MUSICAL: Autofluorescence Super-resolution Microscopy for Molecular Histopathology of Matrix Proteins in Fibrotic Diseases
}

\author{
Biswajoy Ghosh, ${ }^{1}$ Jyotirmoy Chatterjee, ${ }^{1}$ Ranjan Rashmi Paul, ${ }^{2}$ Pooja Lahiri, ${ }^{1}$ \\ Mousumi Pal, ${ }^{2}$ Pabitra Mitra, ${ }^{1}$ Balpreet Singh Ahluwalia, ${ }^{3,4}$ Krishna Agarwal, ${ }^{3 *}$ \\ ${ }^{1}$ Indian Institute of Technology Kharagpur, West Bengal, India \\ ${ }^{2}$ Guru Nanak Institute of Dental Sciences and Research, Kolkata, West Bengal, India \\ ${ }^{3}$ UiT - The Arctic University of Norway, Troms $\varnothing$, Norway \\ ${ }^{4}$ Department of Clinical Science, Intervention and Technology, \\ Karolinska Insitute, Stockholm, Sweden
}

*To whom correspondence should be addressed; E-mail: krishna.agarwal@uit.no.

Fibrosis is an extracellular matrix disease affecting several vital organs' functions and can lead to life-threatening pathologies like cancer. The standard diagnostic protocol involves an immuno-histochemical examination of the fibrosislinked protein's distribution in the tissues. Of specific interest are the primarily affected dense matrix-proteins like collagen. But labeling such dense proteins is prone to subjectivity. Besides distribution, the collagen nano-fibril characteristics, usually revealed by ultra-structural imaging, also have diagnostic relevance. Super-resolution microscopy can aid high-resolution clinical decisions by imaging protein nano-structures but is challenging for dense proteins. Here we exploit the natural fluctuations of tissue autofluorescence (tAF) signal from proteins in dense tissue matrix for super-resolving them. We achieved super-resolution over a clinically relevant large area using a simple, low-cost, low numerical aperture (NA) optical microscope and a recent fluctuation-based nanoscopy technique MUSICAL. We could quantify protein distribution and nano-fibril dimensions as low as $43 \mathrm{~nm}$ in human oral mucosa with pre-cancer fibrosis and mouse skin samples to assess disease progression. Thus tAF-MUSICAL enables early, label-free, and high-resolution diagnosis of matrix-associated diseases like fibrosis. 


\section{Introduction}

The occurrence of several autofluorescent molecules in biological cells and tissues $(1,2)$ has prompted their use for label-free investigations using conventional optical microscopes in several niche areas like cancer diagnosis (3) and regenerative medicine (4). Since optical microscopy is diffraction-limited (DL), the nano-structural details of the molecules cannot be fully realized using them, and it is here that super-resolution microscopy has proved invaluable. Super-resolution microscopy, also popularly called optical nanoscopy, is well established for labeled samples, but its potential for autofluorescence is not well explored (5). The few studies that reported super-resolution imaging using autofluorescence did so for cellular and subcellular structures (6-8), but super-resolution in tissues remains a challenge (5). The challenge in super-resolving tissues arises from the presence of extremely dense tissue matrix proteins such as collagen (connective tissue) and keratin (epithelial tissue) with highly variable local densities $(5,9-11)$. Since matrix proteins provide structural integrity to tissues, any change in these proteins in terms of density, distribution, or structure impacts organ functions. The realization of tissue matrix abnormalities can, therefore, be good indicators of pathological manifestation (12).

Other nano-imaging tools like the electron microscope (EM) and the atomic force microscope (AFM) image matrix proteins (13) with unmatched resolution ( $2 \mathrm{~nm}$ ). However, the unspecific target identification limits visualization of the matrix proteins in the sea of several other biomolecules present in the tissue (9-11). Targetted immunofluorescence-based superresolution is a compelling alternative. However, labeling suffers from constraints like (i) the difference in exact spatial location of the fluorescent probes and the actual molecule $(5-8,14)$ compromise the structural information, (ii) irregularity in the number of fluorescent probes per binding site upon indirect immunostaining compromises density information (5), and (iii) 
underexposed epitopes that are unavailable for probe binding on the target molecules cause incomplete labelling (9). Further, raising antibodies for labeling collagen requires non-denatured collagen due to their large 3-dimensional triple-helical conformation. We, therefore, lose reactivity and specificity substantially in practical settings that involve denatured and fixed tissue sections.

Autofluorescence seems to be ideal for super-resolution imaging $(6,8)$ as it is source-emitted (better localization and fidelity in distribution); therefore, it overcomes labeling limitations. Fluorescence imaging applications like the multi-photon microscopy (MPM) and second harmonic generation (SHG) imaging also use autofluorescence for imaging collagen and keratin $(15,16)$. However these autofluorescence methods for tissue collagen and keratin are diffraction limited. A recent study by Barlow et al. has shown use of the Zeiss Airyscan (a 32-channel GaAsP photomultiplier tube (PMT) array detector (17)) on top of MPM and SHG imaging can improve resolution up by a factor of $1.7 \times(18)$.

Single-molecule localization-based super-resolution methods like PALM, STORM, PAINT have strict requirements on the rate of photokinetics, which manifest as significant spatiotemporal sparsity in fluorescent images. Thus, specialized probes and chemical interventions are mandatory, restricting the usage of a native property like autofluorescence. Also, these methods need long dark states and high photon count for precise localization (5). Hence, imaging time in terms of exposure time per image frame and the total number of image frames needed for a single reconstruction is indefinitely long for tissues with high densities of matrix proteins. Nanoscopy methods like STED, GSD, and RESOLFT are feasible in principle for tissue superresolution because of their point scanning mechanism. These methods have worked in tissues with labeled fluorescence, and with relatively sparse and intracellular targets $(7,8)$, but their application for imaging autofluorescence in dense structures is pending exploration. Furthermore, point-scanning based super-resolution require the microscope to be confocal and limits 
throughput (5).

Visualization of protein density variations across the different tissue zones is key for histopathological examinations. The optical restrictions that most super-resolution methods impose limits the usable thickness of the sample space for imaging to be as low as $500 \mathrm{~nm}$ (for a $100 \times$ objective, 1.4 NA). Such restrictions, in essence, removes information of prominent density variations otherwise seen in microscopic evaluation of histopathological samples of 4-5 $\mu \mathrm{m}$ section thickness. Even if super-resolution methods achieve a wide field-of-view by stitching individual images, it does not integrate the depth information necessary to assess protein density variation and compare pathologies. The multiple signal classification algorithm (MUSICAL) (19) is a super-resolution method that has shown potential in resolving nano-structures with high NA objectives. MUSICAL relies only on fluctuations in fluorescence intensity measured in the camera because of fluctuations in fluorescence emissions irrespective of the mechanism underlying the fluctuations and the density of emitters. Therefore, being a high-density super-resolution method, it is independent of the emitter density of the sample. We adapted this feature of MUSICAL in the high depth of field nanoscopy using a low power objective. A broad field of view is an added advantage of using a low NA objective, enabling visualization across a larger portion of the tissue. The choice of optics and MUSICAL algorithm coupled with the described advantages of autofluorescence makes tAF-MUSICAL ideal for imaging protein variations and nanofibril structures simultaneously. Further, MUSICAL's ability to suppress out-of-focus background (19) enables it to strictly visualize the density of a fixed tissue thickness across the objective's depth of field. Thus, tAF-MUSICAL projects a 'volumetric' density variation, e.g., $450 \times 350 \times 3.7 \mu \mathrm{m}^{3}$ (for a 0.4 NA objective) on a $2 \mathrm{D}$ super-resolved image plane.

Several diseases are associated with changes in matrix proteins. Fibrosis is a pathology of collagen that causes many chronic inflammatory diseases, including systemic sclerosis, glomerulonephritis, interstitial lung disease, non-alcoholic steatohepatitis (NASH), Crohn's disease, 
and heart disease (20). Fibrosis also influences cancer metastasis in the oral cavity, liver, and lungs (21). The study of the tissue matrix is also crucial for wound repair and regenerative medicine as scar tissues are a resultant of fibrosis. We show the role of density variation and nanoscopy features of tAF-MUSICAL using various fibrotic conditions to diagnose the disease progression and state of fibrosis. 


\section{Results}

tAF-MUSICAL approach for optical nanoscopy of tissue autofluorescence. Fig. 1 summarizes the steps involved in generating a tAF-MUSICAL image. We pre-process the samples to minimize interference from moisture and formaldehyde (fixative)-associated fluorescence (22). Collagen and keratin molecules need excitation in the UV region (320-410 nm) to emit autofluorescence in the blue region $(400-510 \mathrm{~nm})(1,2,15,23)$ of the electromagnetic spectra. Thus, we used suitable microscope filter cubes (Supplementary Fig. S1) to collect the desired autofluorescence. The image frames captured on a low NA objective (0.4 NA, 20x) accumulated the desired photokinetic fluctuations as spatial variations in fluorescence intensities across the frames.

For MUSICAL we use microscopy image stacks $(\mathrm{k} \approx 500)$ with a frame-rate of $14 \mathrm{fps}$ with an exposure time of 15-100 ms. MUSICAL, a multi-frame high-density super-resolution method, uses an indicator function $\left(i_{\mathrm{MUSICAL}}\right)$, which is calculated based on microscope optics-driven modeling of signal $(s)$ and noise $(n)$. This modeling in turn involves statistical separation of optical mapping (technically called as point spread function and abbreviated as PSF) of sample structure from noise using singular value decomposition as explained in the following. The value of the indicator function at a point $p$ in the sample region indicates the presence of a fluorescence emitter as:

$$
i_{\mathrm{MUSICAL}}(p)=\left(\frac{s}{n}\right)^{\alpha}
$$

The algorithm calculates the indicator function map sequentially as a square window of size N (matching the spatial spread of the PSF) slides across the image's spatial dimension and stitches them later. The scale factor $\alpha$ controls the contrast in each sliding window and contributes to smoothness across the windows during the stitching operation. For our results, we used $\alpha=4$. Pre-processing involves applying a Gaussian function on the windows, making 
it a soft window that softens the truncation associated artificial noise arising from considering small window sizes at a time by weighing the measured intensities based on the distance of a pixel in the window from the center pixel. Each soft window is represented as an $\mathrm{N}^{2} \times \mathrm{K}$ matrix to perform singular value decomposition towards estimating signal(s) and noise (n). A singular value decomposition (SVD) of the measurement matrix produces eigenvectors with decreasing singular values $(\sigma)$. A manually decided threshold $\left(\sigma_{0}\right)$ at the 'knee' region of a $\log _{10} \sigma$ plot classifies the eigenvectors into the signal vector $\left(\mathbf{Q}_{\mathbf{s}}\right)$ for values above the threshold and remaining as the noise vector $\left(\mathbf{Q}_{\mathbf{n}}\right)$. Additionally, the projection of PSF vector $(\mathbf{P})$, i.e., calculated purely from optical parameters (emission wavelength $\lambda$, microscope magnification $M$, numerical aperture $N A$, and detector pixel size $e$ ) on the signal and noise vectors to give the projection distances, i.e., $s$ and $n$

$$
\begin{array}{r}
s=\left\|\mathbf{P}^{T} \cdot \mathbf{Q}_{\mathbf{s}}\right\| \\
n=\left\|\mathbf{P}^{T} \cdot \mathbf{Q}_{\mathbf{n}}\right\|
\end{array}
$$

For further read on the mathematical derivations, more insight into the variables, and comparison with other optical nanoscopy methods, please refer (19).

\section{tAF-MUSICAL for nano-structural visualization of keratin and collagen fibrils in tissues}

The spatial dimension of microscope images $-1388 \times 1040$ pixels translates to a field of view (FOV) of $448 \times 335 \mu \mathrm{m}$ in the physical space. For a wavelength $(\lambda)=460 \mathrm{~nm}$, refractive index of medium $\left(\mu_{\text {air }}\right)=1, \mathrm{NA}=0.40$, magnification $=20 \times$, and pixel size $(e)=6.45 \mu \mathrm{m}$, the images had a depth of field (DOF) of $\sim 3.7 \mu \mathrm{m}$ as per the following equation ():

$$
D O F=\frac{\lambda \cdot \mu}{N A^{2}}+\frac{\mu}{M \cdot N A} e
$$

The diffraction-limited (DL) image with a high NA (1.4) objective has a much smaller FOV (90 $\left.\times 67 \mu \mathrm{m}^{2}\right)$ and DOF $(0.55 \mu \mathrm{m})$ compared to the low NA DL image. Consequently, the high 
NA image has a higher theoretical resolution of $164 \mathrm{~nm}$ (Abbe's criterion), while a $0.40 \mathrm{NA}$ objective can resolve only up to $575 \mathrm{~nm}$ spatially.

We performed tAF-MUSICAL on the tissue sections with low NA objectives to collect image frames. A small section of a tissue image (Fig. 2 a-c) compares a low NA image with the highest available resolution from a diffraction-limited (DL) epifluorescence microscope, i.e., with a $100 \times, 1.4$ NA objective along with the achieved super-resolution from low NA image frames. The tAF-MUSICAL image, in contrast to DL images, shows nano-fibrillar structures with density variation across the image (Fig. 2 a-c). The full-field tAF-MUSICAL image with structures corroborative to SEM images is available in Supplementary Fig 2. For high NA epifluorescence tissue images, we see that the fine tissue-matrix structures have poor clarity (Fig. 2 a). A possible explanation of here is the very high density of the autofluorescent matrix proteins even at a very thin DOF. We say this since sparser proteins show a much superior clarity/contrast of fine structures at the identical imaging specifications.

We imaged keratin specific skin epidermal (Fig. $2 \mathrm{~d}$ ) and collagen specific skin dermal tissue (Fig. 2 e) using tAF-MUSICAL and visualized the density variations across a small field. The tAF-MUSICAL reconstructed fibril structures of $43 \mathrm{~nm}$ thickness, which corresponds to a 13.4-fold improvement of lateral resolution over the theoretical Abbe's limit (575 nm for $\lambda=460$ nm and NA of 0.40 ). Further, the distribution of the keratin and collagen sizes corresponds to keratin macro-fibrils (24) and collagen fibrils (25) (Fig 2 d,e - insets).

\section{Comparing the performance of AF and labeled fluorescence using tAF-MUSICAL super-}

resolution. We used purified collagen-I fibers and compared the super-resolved structures obtained from tAF-MUSICAL on autofluorescence (blue) (Fig. 3 a,b) and MUSICAL on collagenI specific labeling (red) (Fig. 3 c,d). In full-frame images (Fig. 3. a,c), although both autofluorescence and labeled image appear similar, we noted that the autofluorescence provided more 
prominent density variations of the bundles. Co-localization between diffraction-limited images of autofluorescence and labeled fluorescence presented a similarity of 96-98\% (Supplementary Fig. 3a). On the other hand, tAF-MUSICAL and MUSICAL (on fluorescent label) images exhibit $60-80 \%$ similarity, depending on the region (Fig i-1). Since molecular specificity was not a problem for autofluorescence when imaging purified Collagen-I, the source of difference is the subjectivity of fluorophore labeling, and the spatially shifted molecular localization in labeled fluorescence. A zoomed-in tAF MUSICAL (Fig. 3 b,d) autofluorescence shows a uniform density while in the labeled image, it presents a non-uniform distribution of collagen. Since we purely imaged collagen I from collagen I only sample, the autofluorescence emitterdensity information is fully reliable over fluorescent labeling which shows only the proteins that were successfully labeled (Supplementary Fig S3 c,d), it is understandable that autofluorescence super-resolution provides a superior estimate of collagen density.

We also made a structural comparison between autofluorescence and labeled fluorescence in the collagen-rich animal skin connective tissue (Fig. 3 e-h). The similarity between tAFMUSICAL and MUSICAL (with labeling) images of tissues was 60-80\%, the range being similar to that observed in purified collagen-I samples (Fig. $3 \mathrm{~m}$-p). Thus, we can infer that in the tissue samples, the autofluorescence from other spectrally overlapping autofluorescent sources is rare.

We carefully observed regions in the diffraction limited images of tissues that exhibited significant density variations. For example, in (Fig. 3 f,h), we observe a triangular region of high density in the diffraction limited images. Only in the tAF-MUSICAL mode were we able to delineate the density variation, a feature absent in the MUSICAL (with labeling). The similarity map showed a low match between them in the high-density triangular region (Fig. 3 $\mathrm{n}, \mathrm{p}$ ), which is a manifestation of MUSICAL (with labeling) not being sensitive to the density variation as opposed to tAF-MUSICAL. Thus tAF-MUSICAL provides a reliable display of 
density variation of collagen in tissues. Since, in the case of indirect immunostaining, the number of fluorophores binding to each molecule is subjective, it is less likely to estimate small changes in distribution accurately.

Thus, in terms of molecular specificity and density variations, the tissue autofluorescence super-resolution outperforms the labeled fluorescence super-resolution.

\section{tAF-MUSICAL for imaging and detection of pre-cancer stages. We used tAF-MUSICAL} to image four pre-cancer stages - (Fig. 4 a-e) normal oral mucosa (NOM), (Fig. 4 f-j) oral submucous fibrosis (OSF - benign pre-cancer), (Fig. 4 k-o) OSF with dysplasia (OSFD-the high potential of malignancy), and (Fig. 4 p-t) oral squamous cell carcinoma (OSCC - cancer). The stage-wise clinical diagnosis was performed by pathologists using the HE-stained images (Fig. 4 c,h,m,r)(Supplementary Fig. S5). We employed appropriate histopathological and molecular imaging standards to validate the spatial abundance of the collagen (Fig. 4 d,i,n,s) and keratin (Fig. 4 e,j,o,t) proteins in the epithelium and sub-epithelium. Oral oncopathologists supervised and confirmed all validations. The oral mucosa (Fig. $5 \mathrm{u}$ ) has (i) a keratin-rich epithelium layer and (ii) a collagen-rich sub-epithelium just beneath the epithelium layer. In oral tissues, collagen and keratin are exclusively present in the sub-epithelium (2) and the epithelium (26), respectively. Since these two molecules overlap spectrally in autofluorescence $(2,23)$ but are anatomically differently localized with exclusivity, tAF-MUSICAL can image them simultaneously.

Collagen distributions in the sub-epithelium in tAF-MUSICAL images (Fig. 4 b,g,l,q) matched with the corresponding VG stained sections (collagen-red color) (Fig. 4 d,i,n,s) in all stages of oral pre-cancer. We used the VG stain to image collagen density variations as it stains all collagen types rather than just collagen-I immunostaining that suffers from other subjectivities (Supplementary Fig. S4). The sub-epithelial tissues that well-differentiated with 
distinct papillary (upper layer) and reticular (lower layer) sub-epithelium in NOM (Fig. 4 b,d) showed homogenous distribution in OSF (Fig. 4 g,i). In OSFD, the reticular sub-epithelium showed high density compared to the papillary tissue with peri-vascular fibrosis (Fig. 4 1,n). Due to the disruption of the sub-epithelial integrity in OSCC, its layers were indistinguishable (Fig. 4 q,s).

We found a complete spatial match between epithelial tAF-MUSICAL (Fig. 4 b,g,l,q) and keratin-specific immuno-staining (keratin-brown color) (Fig. 4 e,j,o,t) for all four stages. The normal stratified oral epithelium has high keratin expressing epithelium proper (upper layer) and low keratin expressing basal and supra-basal (lower layers) layers (Fig 4 b, e). However, keratin expression gradually increases and becomes less distinct between two epithelial zones in OSF (Fig 4 g,i) and OSFD (Fig. 4 1,o). In cancer, the donut-shaped keratin peals become prominent (Fig. $4 \mathrm{v}, \mathrm{x}-\mathrm{z}$ ), and the epithelial islands that invaded the sub-epithelium show lower expression of keratin compared to OSFD (Fig. 4 q,t,w).

For quantifications, we divided the epithelium (E) and sub-epithelium (SE) into two distinct zones. We annotated the tissue layers of epithelium proper as the upper epithelium (UE), basal/supra-basal epithelium as the lower epithelium (LE), papillary sub-epithelium as the upper sub-epithelium (USE), and reticular sub-epithelium as the lower sub-epithelium (LSE) in tAF-MUSICAL images. Interestingly, the ratios of keratin and collagen expressing in the epithelium and the sub-epithelium emerged as a prominent distinguishing feature of pre-cancer stages (Fig. 5 a-d) (Supplementary Fig. S6). Realization of this occurred as with advancing stages, the distinction between epithelium and sub-epithelium in TAF MUSICAL declines but again gains some prominence in OSCC (Fig 4 b,g,l,q). We used variance as a measure of tissue density variations in the layers and noted layer-wise measurements to delineate disease stages (Fig. 5 e,j) (Supplementary Fig. S7).

In conclusion, we find that tAF-MUSICAL has the ability to indicate the differential expres- 
sion of the two proteins and deduce pathologically relevant features like matrix accumulation, loss of heterogeneity, peri-vascular fibrosis, hyalinization, epithelial invasion, and keratin pearl formation.

tAF-MUSICAL overcomes problems due to epitope masking. Oral leukoplakia (OLKP) is globally the most prevalent precancer of the mouth (27). Abnormal deposition and condensation of keratin over the mucosal epithelium is a marker of OLKP, resulting in the para-keratinized plaque layer (Fig. 6 a,b). However, our results from keratin immunostaining (Fig. 6b) and prior literature (9-11) suggest that labeling in highly condensed areas like the para-keratinized layer is inconsistent even with keratin-specific antibodies. The patchy labeling is due to modification of the binding site on keratin protein or its condensation, causing the masking of keratin epitopes, rendering it unavailable for labeling. The immunostained OLKP tissue shows almost half the occurrence of keratin found in epithelium-proper due to incomplete labeling in the parakeratinized layer (Fig 6j), which contrarily is heavily keratin-dense (28). As labeling artifacts do not limit tAF-MUSICAL, we observed the para-keratinized layer to have a 2-fold higher keratin expression than epithelium proper (Fig 6 c,i).

In the epithelium (specifically epithelial papilla), the basal and suprabasal layers (lower epithelium LE) are keratin deficient while the epithelium proper (upper epithelium UE) in OLKP is keratin-rich (Fig. 6 b,f,g). Since no epitope masking hinders keratin labeling in the region, this observation matched for tAF-MUSICAL and keratin labeling (Fig 6 h,i,j). Further, we observe a characteristic bundling of subepithelium in the tAF-MUSICAL of OLKP tissue (Fig. 6e) otherwise not seen in OSF or OSFD. In Supplementary Fig. S8 we have shown the layer-wise ratios for all the layers in OLKP.

The differences in the relative expressions of epithelial keratin and sub-epithelial collagen are prominent for the dysplastic case of OLKP (Fig 6d) but inconspicuous for OSFD (Fig 4k). 
Therefore, in addition to identifying precancer stages, the tAF-MUSICAL can be used to delineate the type of precancer occurring in the same anatomy based on differential expression of matrix proteins.

tAF-MUSICAL in pathological fibrosis and post-wound scarring. We explored the applicability of tAF-MUSICAL in (i) pathological fibrosis induced by topical application of arecanut extract on mouse dorsal skin and (ii) non-pathological fibrosis in normal wound scars on mouse dorsal skin (Fig 7 a). We chose arecanut extract (ANE) as the agent to study pathological fibrosis as it is the key cause of the precancer-OSF and causes fibrosis on the skin as well (29-31). We applied ANE for 180 days to mimic pathological fibrosis and study its progression using tAF-MUSICAL. We also applied tAF-MUSICAL on healed scars. Wound healing is a natural response of the body to an injury and ends up in a fibrotic scar tissue with abnormal deposition of collagen.

We observed that the density variations within the skin dermis gradually reduce with the progressive pathological fibrosis in all scales(Fig 7 b-k) (Supplementary Fig S9 a-t). To quantify this, we performed a multi-scale analysis variation from a broad field ROI $\left(90 \times 90 \times 3.7 \mu \mathrm{m}^{3}\right)$ to a minimal field ROI $\left(5 \times 5 \times 3.7 \mu m^{3}\right)$ and found that the variation within the ROIs (Fig 8 a-e) and between multiple such ROIs (Fig $8 \mathrm{f}-\mathrm{j}$ ) decrease with pathological fibrosis. The finding suggests pathological fibrosis causes collagen density to become homogenous within a given volumetric field, especially in late stages of fibrosis (60 days and beyond). A similar observation was found previously in OSF sub-epithelial tissue (Fig 4 g). Further broader ROI fields (Fig 8 k-m) produced better delineation of fibrotic progression over smaller ROI fields (Fig 8 n,o) based on collagen variations. However, tAF-MUSICAL on smaller fields reveal nanostructures as low as $43 \mathrm{~nm}$ (Fig. $8 \mathrm{p}-\mathrm{t}$ ). We measured the distribution of the collagen fiber thickness in the pathologically fibrotic samples and found that with advancing fibrosis, the collagen thickness 
distribution becomes narrow (less variation in thickness) and gets left-shifted (thinner fibrils). The histological and corresponding SEM representations are given in Supplementary Fig. S10.

The tAF-MUSICAL image of 60-day post-injury old scar tissue revealed a different distribution of collagen with an upper scar compartment (USC) having a lower tAF-MUSICAL intensity (Fig 7 1, m) (Supplementary Fig. S9 u-x) than the lower scar compartment (LSC). The USC expressed lower tAF-MUSICAL variation compared to the LSC (Fig 8 w). Further, the USC collagen fibrils were thinner than that of LSC (Fig $8 \mathrm{v}$ ). Although the histogram of the collagen diameters in scar was narrow and left-shifted (Fig $8 \mathrm{u}$ ) compared to normal skin dermis (Fig. 8 p), it was broader than late-pathological fibrosis (beyond 60 days) (Fig. 8 s,t). The thinning and lowering of the variance of collagen thickness in (scar) fibrosis compared to healthy skin tissue using AFM imaging is shown in Supplementary Fig. S11.

\section{Discussion}

tAF-MUSICAL approaches the conventional super-resolution imaging from a perspective other than merely achieving nanometer resolution by mining quantifiable protein density changes in FFPE tissue sections. A broad FOV and thick DOF of a low power objective combined with MUSICAL's ability to resolve nanostructures independent of the number density of fluorescent emitters makes tAF-MUSICAL an exciting tool for label-free histology. tAF-MUSICAL is, therefore, a densitometric nanoscopy that utilizes the reliability of autofluorescence in estimating density and localization of matrix proteins in tissue sections. Further, the label-free nature and requirement of only a basic epifluorescence imaging system add to its appeal.

Labeling associated artifacts in widely used clinical techniques like Immunohistochemistry or immunofluorescence to image distribution of marker proteins suffer, mainly when they target very dense molecules as we demonstrated using purified collagen-I and OLKP tissues. Matrix proteins like collagen and keratin, belong to this class of dense matrix proteins and are often 
associated with several pathologies $(12,26)$. We showed tAF-MUSICAL to resolve matrix proteins and reliably quantify their density variations in tissue sections in oral cancer, pathological fibrosis, and wound healing.

tAF-MUSICAL resolves structures $13 \times$ over the theoretical diffraction limit, i.e., up to 43 $\mathrm{nm}$ using low power microscope objective $(20 \times, 0.40 \mathrm{NA})$. The low power objectives reproduce density changes across a spatial field of $448 \times 335 \mu m^{2}$ and a depth of $3.7 \mu \mathrm{m}$. The tissue pre-processing steps ensure the rapid acquisition of image frames for better acquisition of statistically-independent fluorescent emitters producing more non-zero singular elements $(\sigma)$ or the emitter fluorescence signal for MUSICAL. Further, the pre-processing ensures removal of fixative associated autofluorescence, reducing false positives.

Simultaneous application of tAF-MUSICAL for keratin and collagen was possible due to the spectral overlap of their autofluorescence (23) while being anatomically separated. The feature helped us to image the parallel expression levels of the two proteins separated by the basement membrane (32) and hint cross-talks. As we have seen for both the oral pre-cancers - OSF and OLKP, the ratio quantification of keratin-rich epithelium and collagen-rich sub-epithelium helped to distinguish different pre-cancer types and the stages of a pre-cancer. A possible insight into keratin-collagen cross-talk can be the increased expression of basal/suprabasal keratin expression with fibrosis that primarily targets collagen accumulation in the sub-epithelium. As mechanical stiffness affects cellular behavior and gene expressions (33), the denser collagen of fibrosis right below the epithelium likely affects keratin's epithelial expression.

The use of tAF MUSICAL is valid for both clinical and pre-clinical studies. The pathological fibrosis induced in mouse skin demonstrated the simultaneous loss of variation of density and collagen fibril diameters using tAF-MUSICAL. We could also draw similarities between human OSF and mouse fibrosis based on the sub-epithelial variations, which is attractive as they share common etiology. We compared scars after wound healing and pointed out some exciting 
matrix features that are different from healthy tissue and pathological fibrosis. Using density and collagen fibril dimensions, we distinguished pathological fibrosis from a non-pathological one. Further, the two scar compartments distinguished by tAF-MUSICAL have distinct composition, mechanical properties, and relevance to its stress-management adaptation (34). With this, one needs to also note that although we have shown reasonable structural co-localization and molecular correspondences of matrix proteins collagen and keratin, there may be the possibilities of other rarer autofluorescent molecules like elastin and NADH. The application of MUSICAL for tissues can also be extended for labeled fluorophores. Although the earlier Matlab implementation of the MUSICAL algorithm was not optimized for computational efficiency, a new ImageJ plugin of MUSICAL (35) is available that overcomes this limitation and gives a faster performance.

Thus, with the full range of benefits that tAF-MUSICAL brings to FFPE tissue imaging, the method can be incorporated in histopathological practices in clinics and research of fibrosis .

\section{Methods}

Ethical Considerations. We performed the animal study as per the Institute Animal Ethical Committee (IIT Kharagpur, Kharagpur) regulations following the NIH guidelines. Wound healing study in mice was done under approval \# IE-1/JC-SMST/2.18. Animal studies for ANEinduced pathological fibrosis was done under approval \# IE-7/JC-SMST/2.18. Likewise, human oral pre-cancer and cancer study was approved by the Institute Human Ethics Committee of the source Hospital (GNIDSR, Kolkata, India) under approval \# GNIDSR/IEC/ECC/2015/06. Informed consent was taken from the subjects.

Tissue Samples. We obtained the human oral samples (oral cancer and precancer) after biopsy and clinicopathological validation from GNIDSR, Kolkata, India. Two independent oncopathol- 
ogists evaluated the samples for staging using histopathology. $\mathrm{N}=5$ biopsied and formalin-fixed paraffin embedded specimens were used per group (NOM, OSF, OSFD, OSCC, OLKP). Multiple ROIs $(\mathrm{N} \geq 30)$ was collected from each group. We procured commercially available purified collagen-I derived from rat tail (Cat No.C7661, Sigma-Aldrich). For making scar tissues, we created skin full-thickness wounds ( $\sim 4 \mathrm{~mm}$ diameter) on the dorsum of the mouse (male, age-2 months, Swiss albino, weight 30-40 gm) and allowed them to heal for a month till a scar was distinctly visible. We extracted the scar by excision and fixed the samples for further processes. For the creation of chemically induced fibrosis, we used arecanut extract (ANE) — a potent fibrotic agent that was applied on the mouse skin $(2.5 \% \mathrm{w} / \mathrm{v}$ in $50 \%$ ethyl alcohol) every day for up to 120 days. We extracted the fibrotic skin tissue at different time intervals by excision for further processing. We prepared the arecanut extract in-house using locally available arecanut. We baked the nuts at $45^{\circ} \mathrm{C}$ for 1 hour, finely crushed them up to 2-3 mm, and finally solvent extracted them with $50 \%$ methanol for 24 hours. We filtered the insoluble components using a Whatman filter paper No. 1. We finally obtained the powdered arecanut extract after removing the methanol by rotary evaporator (water bath temperature of $50{ }^{\circ} \mathrm{C}$ ) and the water by freeze-drying. $\mathrm{N}=5$ animals were used in each group of arecanut fibrosis (Normal, F18, F30, F60, F180) and scar tissue. Multiple ROIs ( $\geq 30)$ was collected from each group.

Sample Fixation and Processing for tAF-MUSICAL. We submerged the tissue samples in $10 \%$ phosphate-buffered formalin for tissue fixation for 24 hours. We vacuum-embedded the fixed tissues in melted paraffin to obtain tissue blocks. We cut the formalin-fixed paraffinembedded (FFPE) tissue into thin sections of $5 \mu \mathrm{m}$ and mounted them on glass slides coated with poly-L-lysine ( $0.1 \%$ w/v in water, Cat. No. P8920, Sigma Aldrich, Germany). We then removed the paraffin by incubating slides dipped in warm $\left(60^{\circ} \mathrm{C}\right)$ xylene for 30 minutes, followed by immersion of the slides in $100 \%$ ethanol for 5 minutes. We performed antigen retrieval on 
the sections using Tris-EDTA buffer (pH 9.0) using EZ-Retriever System V.2 (BioGenex) with the standard microwave protocol. We dehydrated the samples in graded alcohol $(50 \%$ and $70 \%$, 5 minutes each) up to $100 \%, 45$ minutes followed by air-drying them overnight, and 60-minute oven-baking at $45^{\circ} \mathrm{C}$ before microscopy.

Tissue Staining. For immunostaining (IHC and IF), after antigen retrieval, we incubated the FFPE tissues with primary antibodies (monoclonal anti-collagen-I, Cat No. ab6308, Abcam; monoclonal anti-Pan-keratin, No. 4545, Cell Signaling Technology) overnight at $4{ }^{\circ} \mathrm{C}$ with the factory recommended dilutions. We chose a red fluorescent probe (Alexa Fluor 594) for visualizing labeled collagen-I to measure colocalization with collagen autofluorescence in the blue emission spectra. For IHC, we used horseradish peroxidase-conjugated secondary antibodies using the chromogen 3,3'diaminobenzidine (DAB) for pan-keratin staining. We performed nuclear counterstaining using Hematoxylin in all the IHC slides. For clinical validation and staging of precancer/cancer, we used the Hematoxylin and eosin(HE) stained slides. For staining the subepithelial connective tissue, we performed the van Giesons stain (VG) (differentially stains collagen and muscle fibers in red and other components in yellow) with Hematoxylin nuclear counterstaining.

Imaging Parameters. We imaged the tissue sections with an inverted wide-field epifluorescence microscope (Axio Observer.Z1, Zeiss, Germany)) under a $20 \times$ objective (NA=0.40, Zeiss, Germany). We captured about 500 frames with an exposure time of 20-30 ms and a frame rate of $14 \mathrm{fps}$ to acquire the emission fluctuations from the autofluorescent proteins. The CCD camera (AxioCam MRm, Zeiss, Germany) had a square pixel size of $6.45 \mu \mathrm{m}$. We saved the image frames in a non-compressed OME-TIFF format for MUSICAL. We illuminated the samples using a mercury plasma arc-discharge lamp (X-Cite Series 120 Q, Excelitas Technologies, NYC, USA), having excitation/emission spectra filter cubes (Chroma Technology, Bellows 
Falls, VT). For detecting collagen autofluorescence, we used excitation (ex) and emission (em) peak of $\lambda_{e x}=359 \mathrm{~nm}$ and $\lambda_{e m}=461 \mathrm{~nm}$, and for Alexa Fluor 594 labeled fluorescence, we used $\lambda_{e x}=592 \mathrm{~nm}, \lambda_{e m}=614 \mathrm{~nm}$. For ground truth measurement to establish super-resolution, we imaged the same region of the tissue at the maximum epifluorescence resolution i.e., $100 \times$ objective (oil) and NA=1.4.

Color Deconvolution. We performed the DAB and Hematoxylin color deconvolution in ImageJ using the plugin developed by Gabriel Landini available at https://imagej.net/ Colour_Deconvolution.

MUSICAL. We used the Matlab source code of MUSICAL developed by Agarwal for generating MUSICAL results. The codes are available at the link (https://sites.google. com/site/uthkrishth/musical). We used a sub-pixelation of 10, i.e., the $10 \times 10 \mathrm{MU}-$ SICAL pixels correspond to one physical camera pixel. As suggested previously, we chose the threshold for each image heuristically close the knee of singular value plots. The parameter $\alpha$ was assigned the default value of 4 .

Co-localization of labeled collagen fluorescence vs. collagen autofluorescence. We performed MUSICAL for both autofluorescence and labeled fluorescence of Collagen-I and measured the structural similarity using SSIM (structural similarity index) in MATLAB R2019a.

Image and statistical Analyses. ImageJ line tool was used to determine fibril-thickness from tAF-MUSICAL images with a spatial field of $5 \times 5 \mu \mathrm{m}^{2}$ to clearly visualize fibrils. A total of N=300 fibrils per group was used to generate a histogram plotted in GraphPad Prism 6.0. For the measurement of intensity and variance in oral pre-cancer layers, random patches were generated in specific regions/layers of the tissue to be measured. Intensity ratios were measured 
by generating all the combinations of intensities between the two participant layers $(\mathrm{N}=900)$. For every paired comparisons, paired 2-tailed t-test was used to assess p-value. For multi-group comparisons, ANOVA with Tukey's modification was used and the p-value was measured. For multi-scale analysis for every window size, pixel patches of $300 \times 300$ ( $\mathrm{N}=30$ per group) was used. The measurements of keratin from stained OLKP samples, after color deconvolution, the DAB component was inverted and converted to 8-bit gray scale format before generating patches and quantifying the stain uptake.

Ultra-structure imaging For SEM, the samples were coated with gold-platinum alloy mist and imaged under SEM (MERLIN, Zeiss, Germany) up to 200,000× EM magnification to image the typical collagen fibril structures. For AFM, we imaged the tissue sections under Bruker Multimode 8 Atomic Force Microscope system (Bruker Corporation, USA) imaged the tissue sections in the PeakForce QNM (PF-QNM, Bruker Corporation, USA) mode. 


\section{Conclusion}

We elaborate tAF-MUSICAL_a label-free super-resolution method that obtains nanostructural details and density variation across a broad field of dense tissue matrix proteins. tAFMUSICAL uses low power objectives, protein autofluorescence, and the MUSICAL nanoscopy algorithm to resolve collagen and keratin fibril structures up to $43 \mathrm{~nm}$ along with quantitative volumetric density in tissues. We applied tAF-MUSICAL to extract pathologically relevant features in pre-cancer staging, pathological fibrosis, and post-wound scars. Since several diseases are associated with tissue matrix integrity and distribution, translation of tAF-MUSICAL to histopathology can add value to the arsenal of existing pathological practices for label-free molecular diagnosis.

\section{References}

1. Croce, A. C. \& Bottiroli, G. Autofluorescence spectroscopy and imaging: a tool for biomedical research and diagnosis. European journal of histochemistry: EJH 58 (2014).

2. Monici, M. Cell and tissue autofluorescence research and diagnostic applications. Biotechnology annual review 11, 227-256 (2005).

3. Luo, X. et al. Accuracy of autofluorescence in diagnosing oral squamous cell carcinoma and oral potentially malignant disorders: a comparative study with aero-digestive lesions. Scientific reports 6, 29943 (2016).

4. Vielreicher, M. et al. Taking a deep look: modern microscopy technologies to optimize the design and functionality of biocompatible scaffolds for tissue engineering in regenerative medicine. Journal of the Royal Society Interface 10, 20130263 (2013). 
5. Sahl, S. J., Hell, S. W. \& Jakobs, S. Fluorescence nanoscopy in cell biology. Nature reviews molecular cell biology 18, 685 (2017).

6. Bierwagen, J. et al. Far-field autofluorescence nanoscopy. Nano letters 10, 4249-4252 (2010).

7. Kaufmann, R., Müller, P., Hausmann, M. \& Cremer, C. Imaging label-free intracellular structures by localisation microscopy. Micron 42, 348-352 (2011).

8. Dong, B. et al. Superresolution intrinsic fluorescence imaging of chromatin utilizing native, unmodified nucleic acids for contrast. Proceedings of the National Academy of Sciences 113, 9716-9721 (2016).

9. Asch, B. B. \& Asch, H. L. A keratin epitope that is exposed in a subpopulation of preneoplastic and neoplastic mouse mammary epithelial cells but not in normal cells. Cancer research 46, 1255-1262 (1986).

10. Hendrix, M., Hay, E. D., von der Mark, K. \& Linsenmayer, T. F. Immunohistochemical localization of collagen types $\mathrm{i}$ and ii in the developing chick cornea and tibia by electron microscopy. Investigative ophthalmology \& visual science 22, 359-375 (1982).

11. Mauger, A. et al. Immunofluorescent localization of collagen types i and iii, and of fibronectin during feather morphogenesis in the chick embryo. Developmental biology 94, 93-105 (1982).

12. Morris, B. A. et al. Collagen matrix density drives the metabolic shift in breast cancer cells. EBioMedicine 13, 146-156 (2016). 
13. O'leary, L. E., Fallas, J. A., Bakota, E. L., Kang, M. K. \& Hartgerink, J. D. Multihierarchical self-assembly of a collagen mimetic peptide from triple helix to nanofibre and hydrogel. Nature chemistry 3, 821-828 (2011).

14. Deschout, H. et al. Precisely and accurately localizing single emitters in fluorescence microscopy. Nature methods 11, 253 (2014).

15. Zipfel, W. R. et al. Live tissue intrinsic emission microscopy using multiphoton-excited native fluorescence and second harmonic generation. Proceedings of the National Academy of Sciences 100, 7075-7080 (2003).

16. Campagnola, P. J. et al. Three-dimensional high-resolution second-harmonic generation imaging of endogenous structural proteins in biological tissues. Biophysical journal $\mathbf{8 2}$, 493-508 (2002).

17. Huff, J. et al. The new $2 \mathrm{~d}$ superresolution mode for zeiss airyscan. Nature Methods $\mathbf{1 4}$, 1223-1223 (2017).

18. Barlow, A. M., Mostaço-Guidolin, L. B., Osei, E. T., Booth, S. \& Hackett, T.-L. Super resolution measurement of collagen fibers in biological samples: Validation of a commercial solution for multiphoton microscopy. Plos one 15, e0229278 (2020).

19. Agarwal, K. \& Macháň, R. Multiple signal classification algorithm for super-resolution fluorescence microscopy. Nature communications 7, 1-9 (2016).

20. Wynn, T. A. et al. Common and unique mechanisms regulate fibrosis in various fibroproliferative diseases. The Journal of clinical investigation 117, 524-529 (2007).

21. Piersma, B., Hayward, M. \& Weaver, V. M. Fibrosis and cancer: A strained relationship. Biochimica et biophysica acta. reviews on cancer 1873, 188356-188356 (2020). 
22. ERÄNKÖ, O. The practical histochemical demonstration of catecholamines by formaldehyde-induced fluorescence. Journal of the Royal Microscopical Society 87, 259276 (1967).

23. Wu, Y. \& Qu, J. Y. Autofluorescence spectroscopy of epithelial tissues. Journal of biomedical optics 11, 054023 (2006).

24. Bornschlögl, T. et al. Keratin network modifications lead to the mechanical stiffening of the hair follicle fiber. Proceedings of the National Academy of Sciences 113, 5940-5945 (2016).

25. Bancelin, S. et al. Determination of collagen fibril size via absolute measurements of second-harmonic generation signals. Nature communications 5, 1-8 (2014).

26. Moll, R., Divo, M. \& Langbein, L. The human keratins: biology and pathology. Histochemistry and cell biology $\mathbf{1 2 9}, 705$ (2008).

27. Petti, S. Pooled estimate of world leukoplakia prevalence: a systematic review. Oral oncology 39, 770-780 (2003).

28. Staines, K. \& Rogers, H. Oral leukoplakia and proliferative verrucous leukoplakia: a review for dental practitioners. British dental journal 223, 655-661 (2017).

29. Chiang, M.-H. et al. Characterization of a novel dermal fibrosis model induced by areca nut extract that mimics oral submucous fibrosis. Plos one 11, e0166454 (2016).

30. Pant, I., Rao, S. G. \& Kondaiah, P. Role of areca nut induced jnk/atf2/jun axis in the activation of $\operatorname{tgf}-\beta$ pathway in precancerous oral submucous fibrosis. Scientific reports $\mathbf{6}$, 34314 (2016). 
31. Pant, I., Kumar, N., Khan, I., Rao, S. G. \& Kondaiah, P. Role of areca nut induced tgf$\beta$ and epithelial-mesenchymal interaction in the pathogenesis of oral submucous fibrosis. PloS one 10, e0129252 (2015).

32. Das, R. K. et al. Epithelio-mesenchymal transitional attributes in oral sub-mucous fibrosis. Experimental and molecular pathology 95, 259-269 (2013).

33. Huang, X. et al. Matrix stiffness-induced myofibroblast differentiation is mediated by intrinsic mechanotransduction. American journal of respiratory cell and molecular biology 47, 340-348 (2012).

34. Ghosh, B., Mandal, M., Mitra, P. \& Chatterjee, J. Structural mechanics modeling reveals stress-adaptive features of cutaneous scars. Biomechanics and modeling in mechanobiology 1-7 (2020).

35. Acuña, S., Ströhl, F., Opstad, I. S., Ahluwalia, B. S. \& Agarwal, K. Musij: an imagej plugin for video nanoscopy. Biomedical optics express 11, 2548-2559 (2020).

\section{Acknowledgments}

Funding: SERB, Dept. of Science and Technology Government of India (\# SB/S5/AB-01/2016) funded Biswajoy Ghosh. The time contributed by Krishna Agarwal was supported by Marie Skłodowska-Curie Actions Individual Fellowship project (\#749666) and European Research Council Starting Grant project (\#804233). Balpreet Singh Ahluwalia acknowledges the funding from the Research Council of Norway (project \# NANO 2021 - 288565), (project \# BIOTEK 2021 - 285571). We acknowledge Biorender web tool which was used to make representative diagrams of skin and oral mucosa under academic license. 


\section{Author contributions}

BG and KA designed the study and written the paper.BG performed the experiments, optical microscopy, sample processing, validation studies, animal experiments, human sample experiments, staining, SEM, AFM were performed by BG. MUSICAL was originally designed and was optimized for tAF by KA and implemented by BG and KA. All figures were made by BG. RRP and MP provided the oral precancer and cancer samples as well as provided relevant clinical inputs. Keratin stain was performed by BG and PL. BSA provided inputs relevant to optics and microscopy. JC and PM provided inputs in the overall structure of the manuscript from the biological and analysis perspective. The paper was reviewed by all authors.

\section{Data availability statement}

All data can be availed upon request. The link to MUSICAL and its application is provided in the Methods section. 


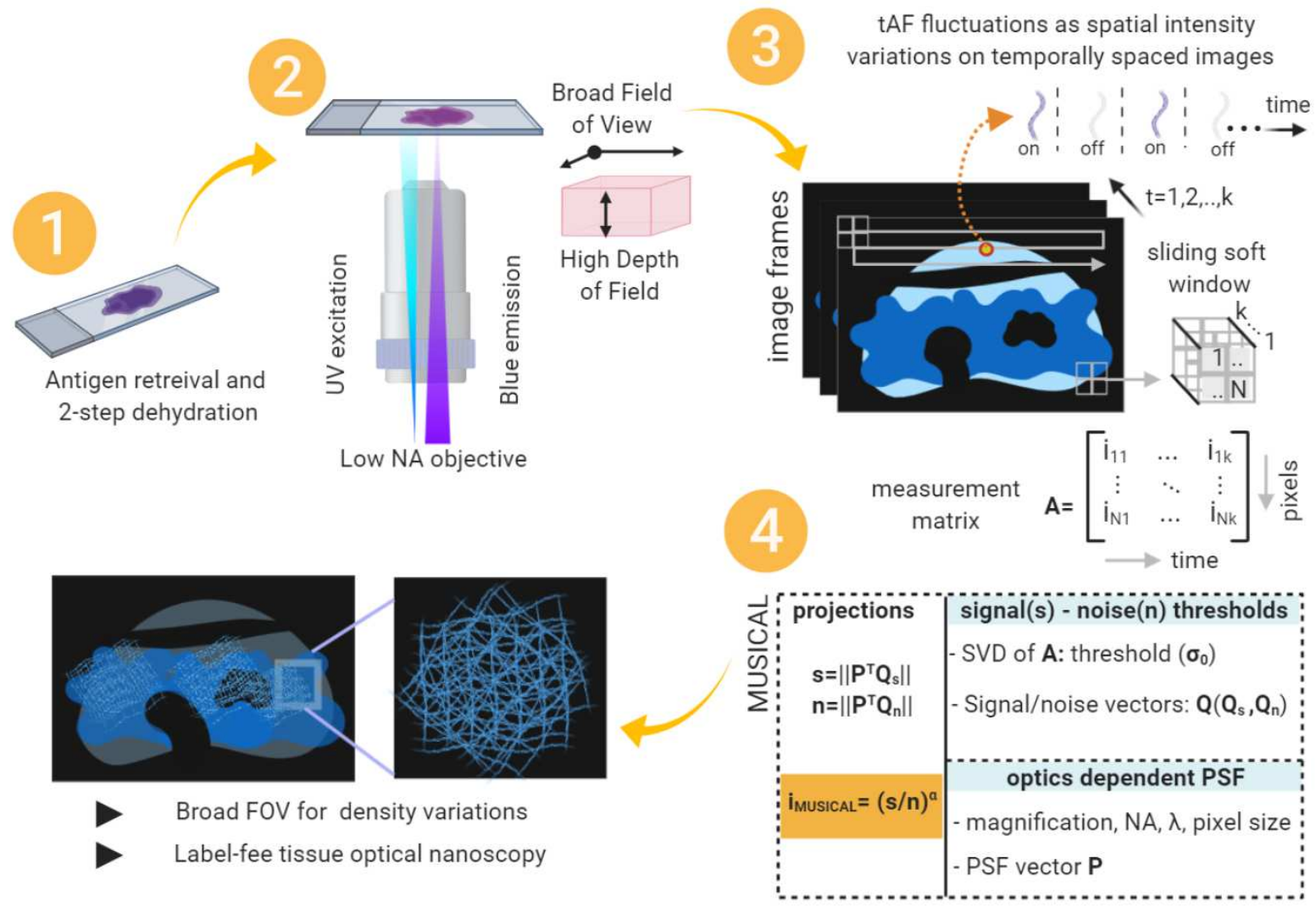

Figure 1: Schematic diagram for tAF-MUSICAL workflow The FFPE tissue sections replete with autofluorescing matrix proteins are mounted on glass slides, deparaffinised and completely dehydrated. Antigen retrieval is performed to remove unwanted fixative-linked fluorescence. A low NA epifluorescence microscope $(20 \times, 0.40$ NA objective $)$ that allows a broad field of view (FOV) of $\sim 323 \times 435 \mu \mathrm{m}^{2}$ and a thicker depth of field (DOF) of $\sim 3.7 \mu \mathrm{m}$ is used to acquire image stacks at excitation and emission wavelengths specific to matrix protein autofluorescence. The rapidly acquired image stacks are processed by MUSICAL to achieve super-resolution as shown and previously described (19). The tAF-MUSICAL image presents density variations on a broad field due to accumulated autofluorescence of high DOF. The tAF-MUSICAL image can be zoomed in for visualizing fibril nano-structures with dimensions as low as $\sim 43 \mathrm{~nm}$. 

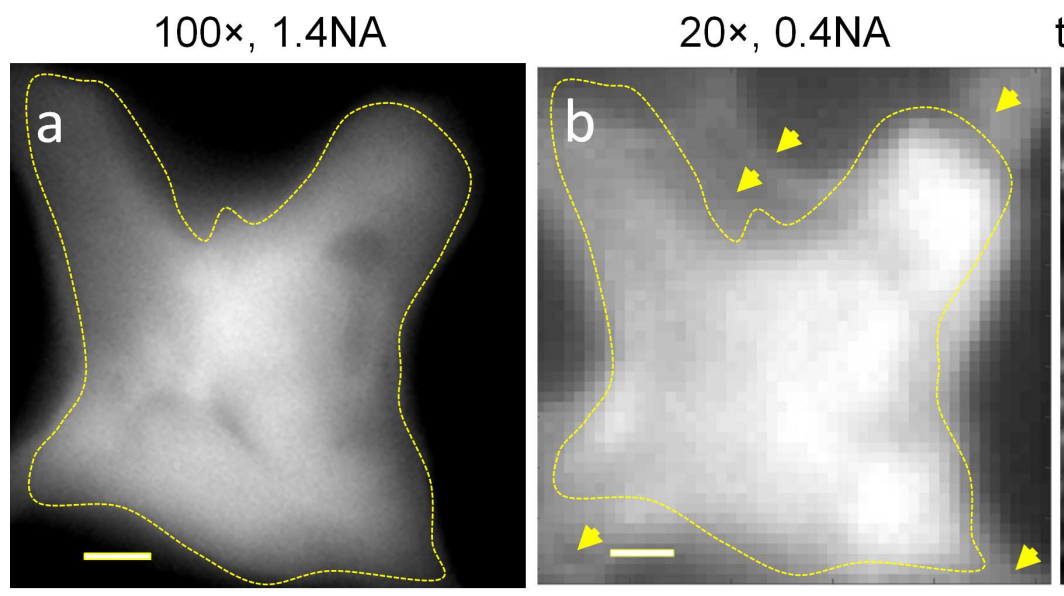

tAF-MUSICAL $(20 \times, 0.4 \mathrm{NA})$
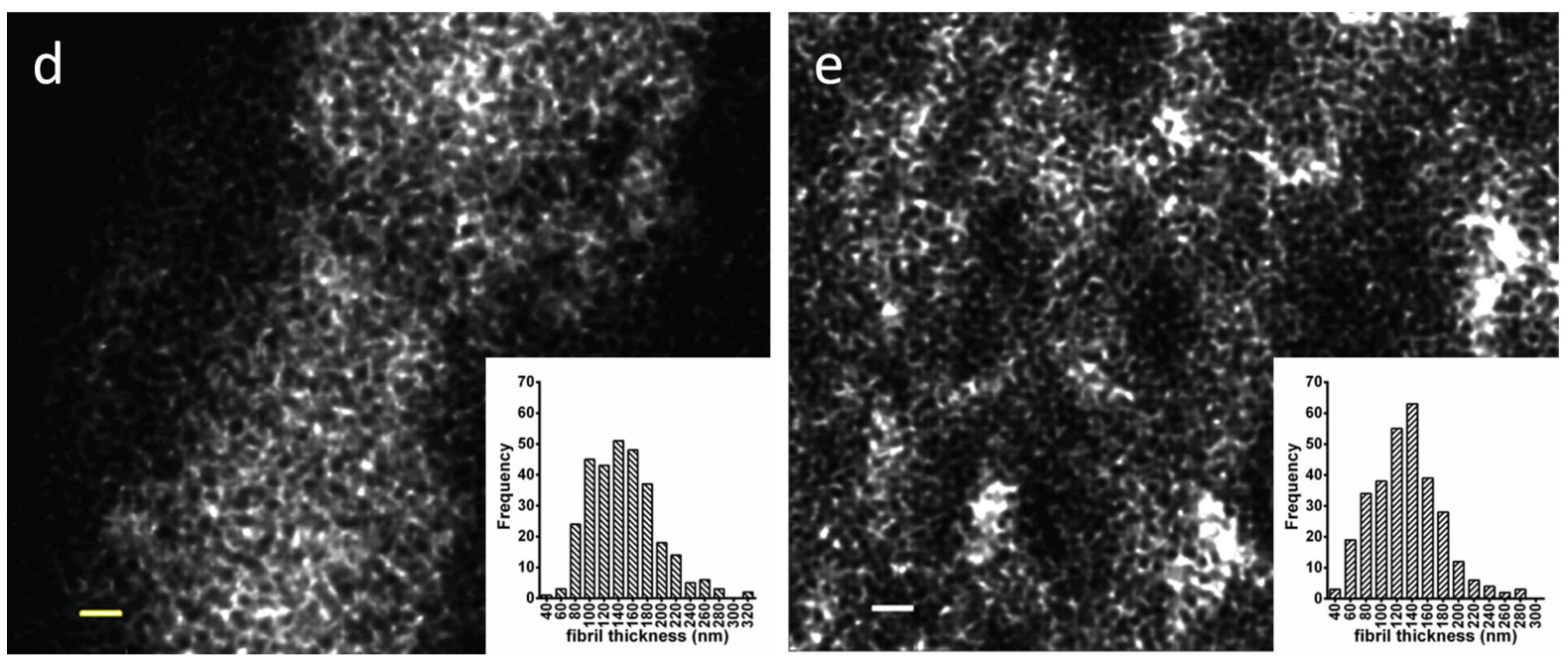

Figure 2: Comparing tAF-MUSICAL super-resolution with diffraction-limited tissue autofluorescence imaging. A region from skin tissue imaged with (a) a $1.40 \mathrm{NA}$ (oil) $100 \times$ objective and (b) a $0.40 \mathrm{NA}, 20 \times$ objective on an epifluorescence microscope. (c) tAF-MUSICAL super-resolution obtained from image frames collected by a $20 \times, 0.4$ NA epifluorescence microscope objective; yellow arrows show exclusive regions in low NA objective, yellow dotted line is the high NA object's region boundary (d) tAF-MUSICAL image region showing keratin rich-epidermal layer of mouse skin tissue. (e) tAF-MUSICAL image region showing collagen rich-dermis of mouse skin tissue. In (d) and (e), inset shows the distribution of respective fibril diameters. scale bar $=2 \mu m$. 

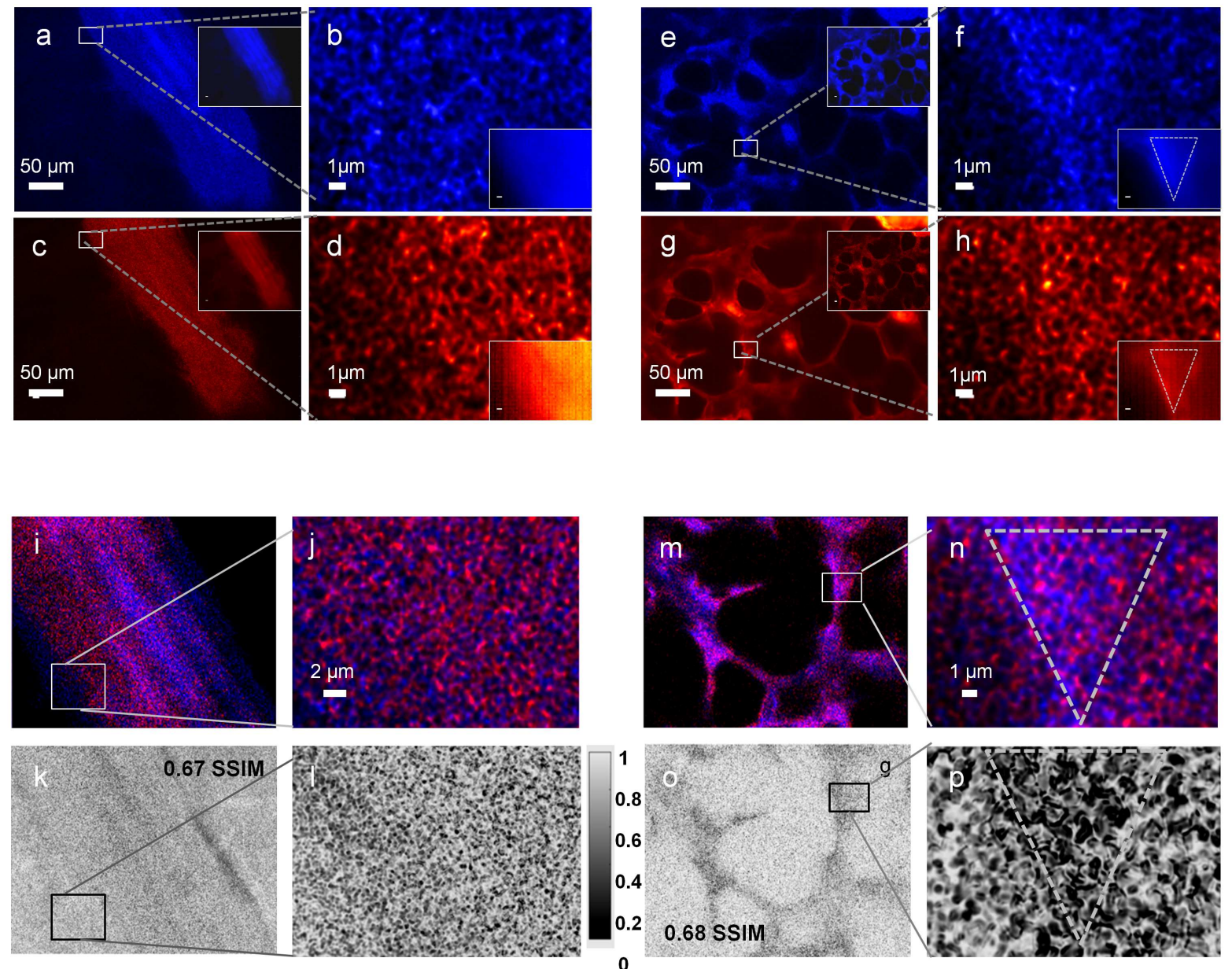

Figure 3: Molecular specificity of tAF-MUSICAL in detecting collagen. MUSICAL superresolution images of (a-d) purified collagen-I and (e-h) skin tissue section imaging. Images derived from (a,b,e,f) tissue autofluorescence (tAF) in blue emission, $(\mathrm{c}, \mathrm{d}, \mathrm{g}, \mathrm{h})$ same tissue immunolabeled with collagen-I and fluorescent probe having red emission. All insets show the corresponding source (diffraction-limited) images $(20 \times, 0.4$ NA objective). (a,c,e,g) are full-field MUSICAL images and (b,d,f,h) are a small region illustrating super-resolved structures. Colocalization of MUSICAL structures of $(i, j)$ pure Collagen-I and $(m, n)$ tissue from auto (blue) and labeled (red) fluorescence. Similarity maps of MUSICAL structures in the red and blue colors images in $(i, j)$ are shown in $(k, l)$ for Collagen-I, and similarly similarity maps of the structures in red and blue colored images in $(m, n)$ are shown in $(o, p)$ for the skin tissue. 


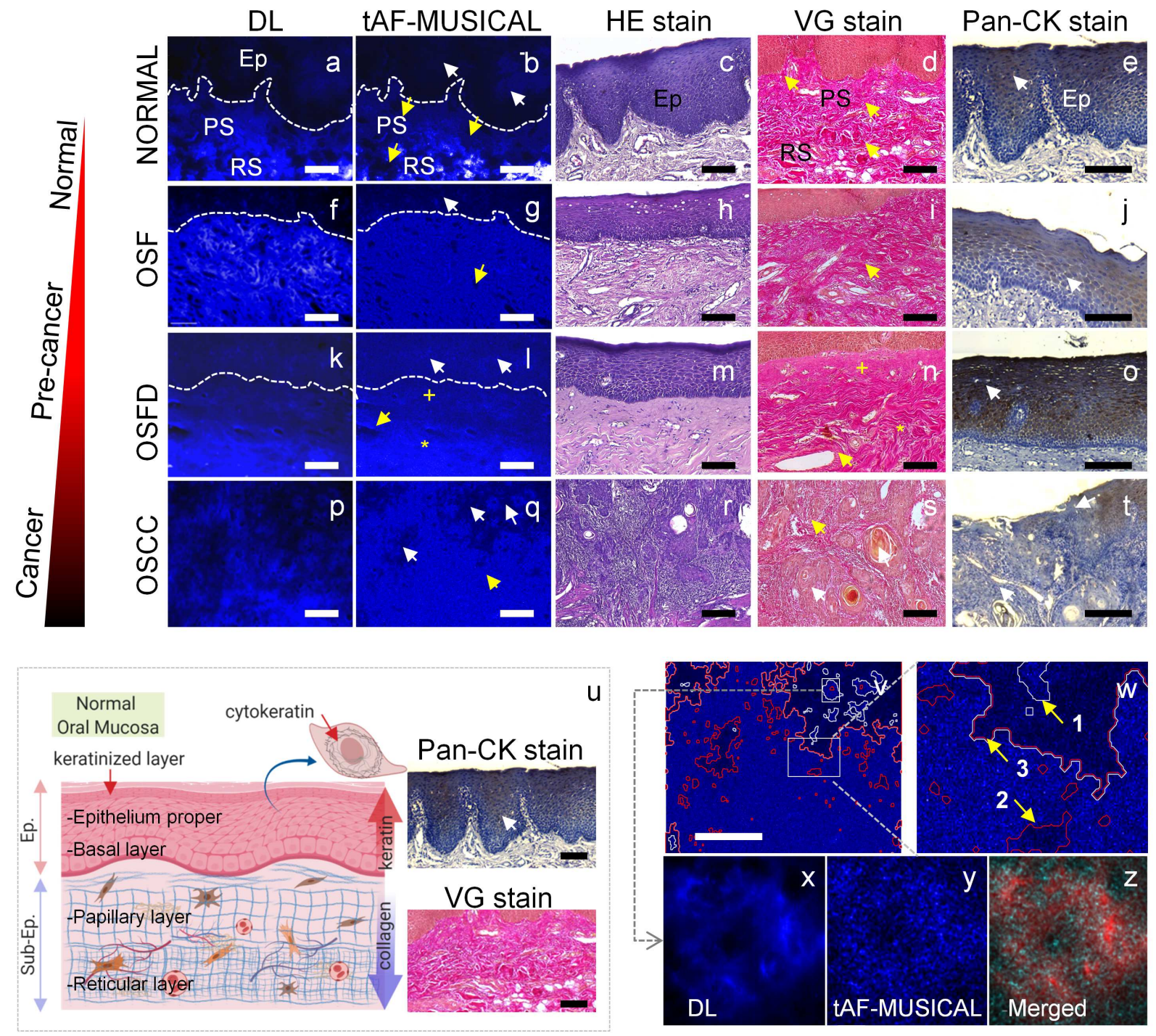

Figure 4: tAF-MUSICAL in imaging pre-cancer progression in the oral mucosa. Corresponding diffraction-limited (DL) image, tAF-MUSICAL, Hematoxylin and Eosin (HE) stained (gold standard), van Gieson (VG) stained (for all collagen in sub-epithelium), and pan-keratin (for all keratin in epithelium) stained sections of (a-e) normal oral mucosa (NOM), (f-j) oral submucous fibrosis (OSF), (k-o) OSF with dysplasia (OSFD), (p-t) oral squamous cell carcinoma (OSCC). The yellow arrows and markers indicate sub-epithelial changes in tAF-MUSICAL corroborative with ground truth images; the white arrows mark groud truth matching epithelial changes. (u) the anatomical structure of oral mucosa includes keratin and collagen's localization (with stained images for reference) in different layers of epithelium and sub-epithelium. (v) tAF-MUSICAL of OSCC with the invasions of epithelial tissues in sub-epithelium,(w) an ROI of OSCC showing: epithelial islands in the subepithelium (red line), keratin pearls within the epithelium (white line) and the non-disrupted basement membrane (red-epithelial side, white - sub-epithelial side). (x,y,z) The DL, tAF-MUSICAL, and merged image of a keratin pearl (a mark of carcinoma). Ep = epithelium, Sub-Ep = sub-epithelium, PS=papillary sub-epithelium, $\mathrm{RS}=$ reticular sub-epithelium. scale bars $=50 \mu \mathrm{m}$ 


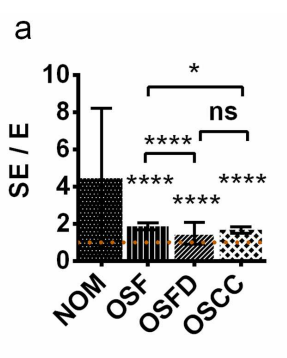

b

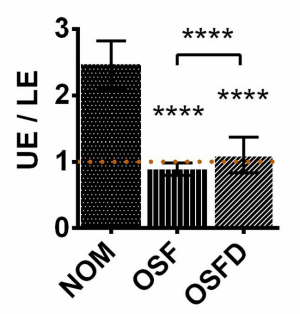

C

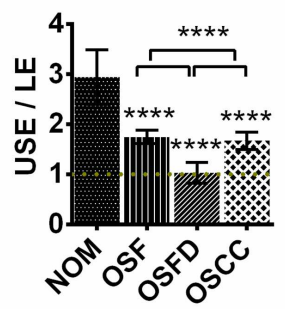

d

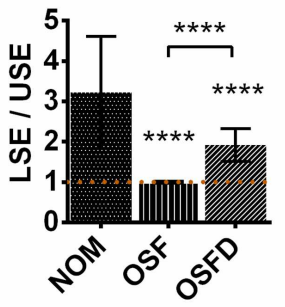

e
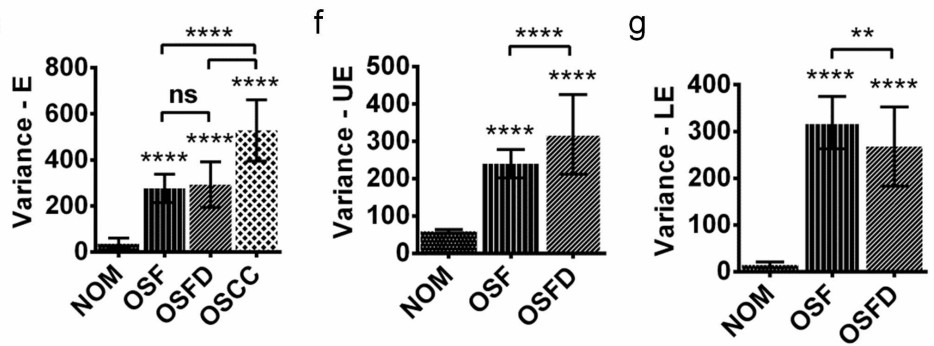

h
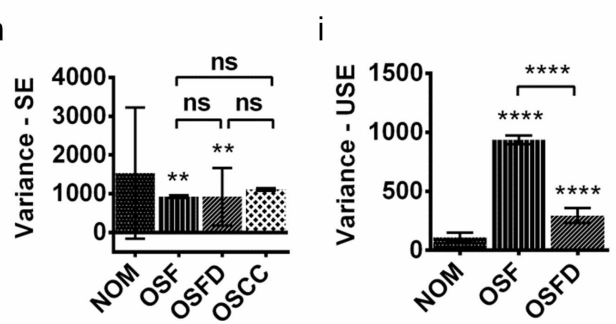

j

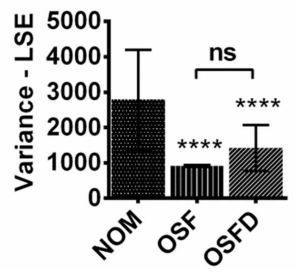

Figure 5: Quantification of broad-field tAF-MUSICAL features in delineating oral precancer stages. (a-d) ratio of intensity between the layers and sub-layers of epithelium and sub-epithelium to delineate the stages-NOM, OSF, OSFD, and OSCC. (e-j) variance quantification of different layers and sub-layers of epithelium and sub-epithelium to delineate the oral pre-cancer stages. In OSCC tissues, the epithelium and sub-epithelium cannot be differentiated due to metastasis and hence were not included in (b,d,f,g,i,j). E-epithelium whole, UE-upper epithelium, LE-lower epithelium, SE-sub-epithelium whole, USE-upper subepithelium, LSE-lower sub-epithelium. UE, LE, USE, LSE of tAF-MUSICAL histologically represent epithelium proper, basal/supra-basal epithelium, papillary sub-epithelium and reticular sub-epithelium respectively. $\mathrm{N}=30$ per group, All plots are represented as $\mu \pm \mathrm{SD}$, ns (not significant) $\mathrm{p}>0.05, * \mathrm{p} \leq 0.05, * * \mathrm{p} \leq 0.01$, *** $\mathrm{p} \leq 0.001$, **** $\mathrm{p} \leq 0.0001$. For statistical analysis we used one-way ANOVA (95\% confidence interval) followed by Tukey's post hoc test. 


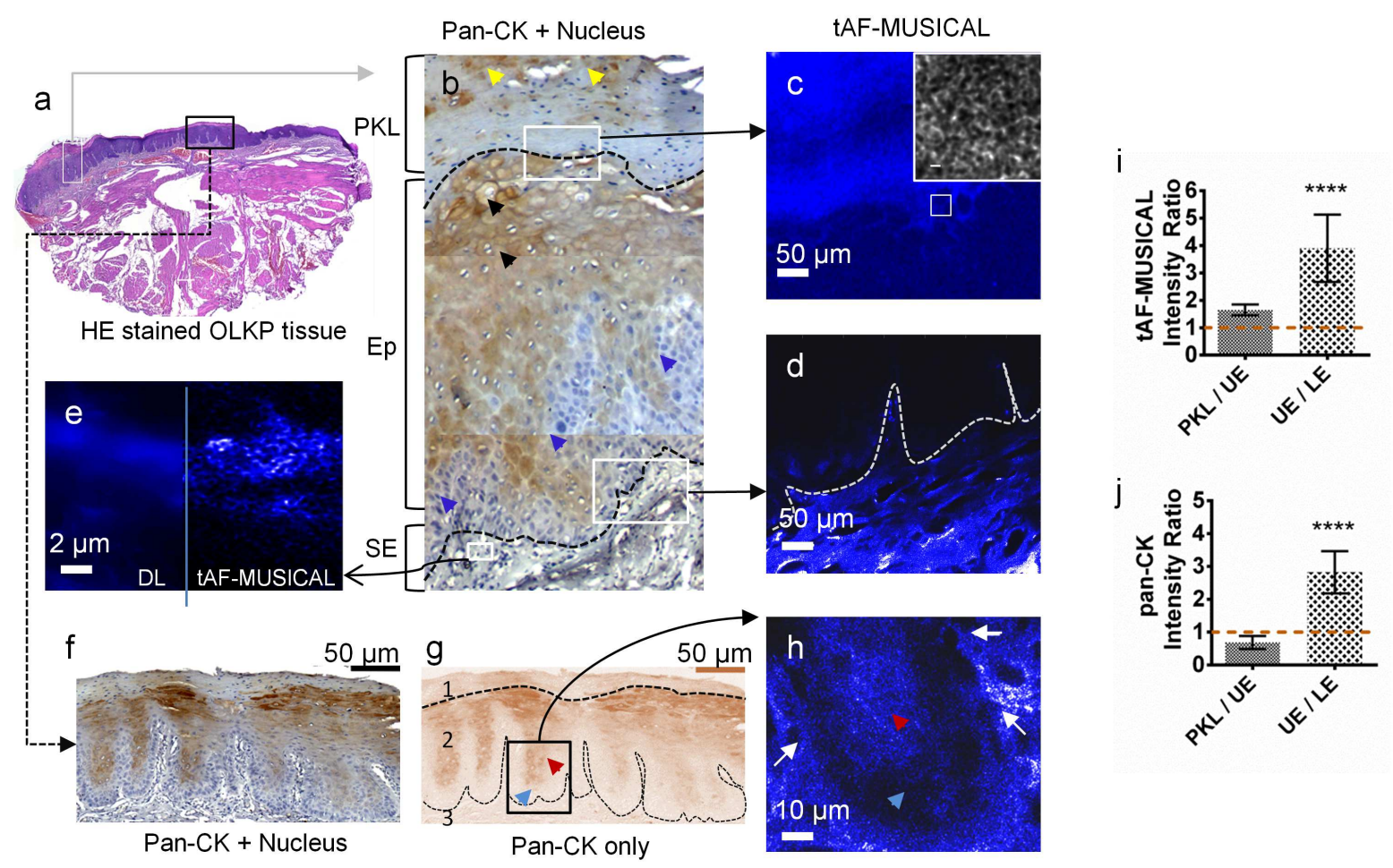

Figure 6: tAF-MUSICAL in detecting keratin associated oral leukoplakia. MUSICAL super-resolution images of (a-d) purified collagen-I and (e-h) skin tissue section imaging. Images derived from (a,b,e,f) tissue autofluorescence (tAF) in blue emission, (c,d,g,h) same tissue immunolabeled with collagen-I and fluorescent probe having red emission. All insets show the corresponding source (diffraction-limited) images $(20 \times, 0.4$ NA objective). (a,c,e,g) are full-field MUSICAL images and $(b, d, f, h)$ are a small region illustrating super-resolved structures. $\mathrm{N}=30$ per group, All plots are represented as $\mu \pm \mathrm{SD}, * * * * \mathrm{p} \leq 0.0001$. For statistical analysis we used two-tailed t-test (95\% confidence interval). 


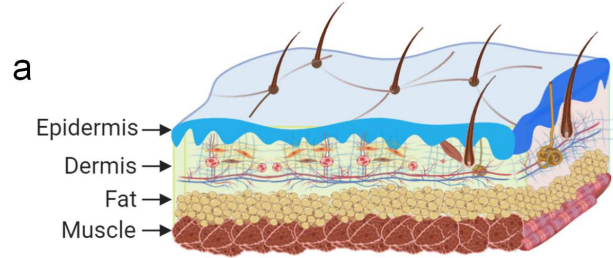

Healthy skin

(mouse)

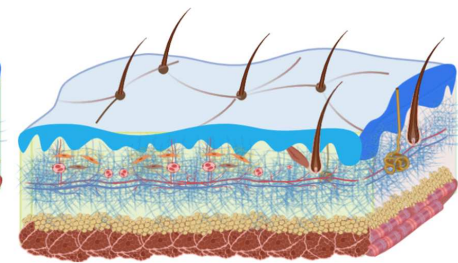

Arecanut-induced (pathological) fibrosis

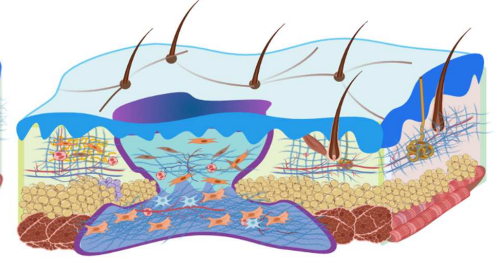

Post-wound

scar tissue
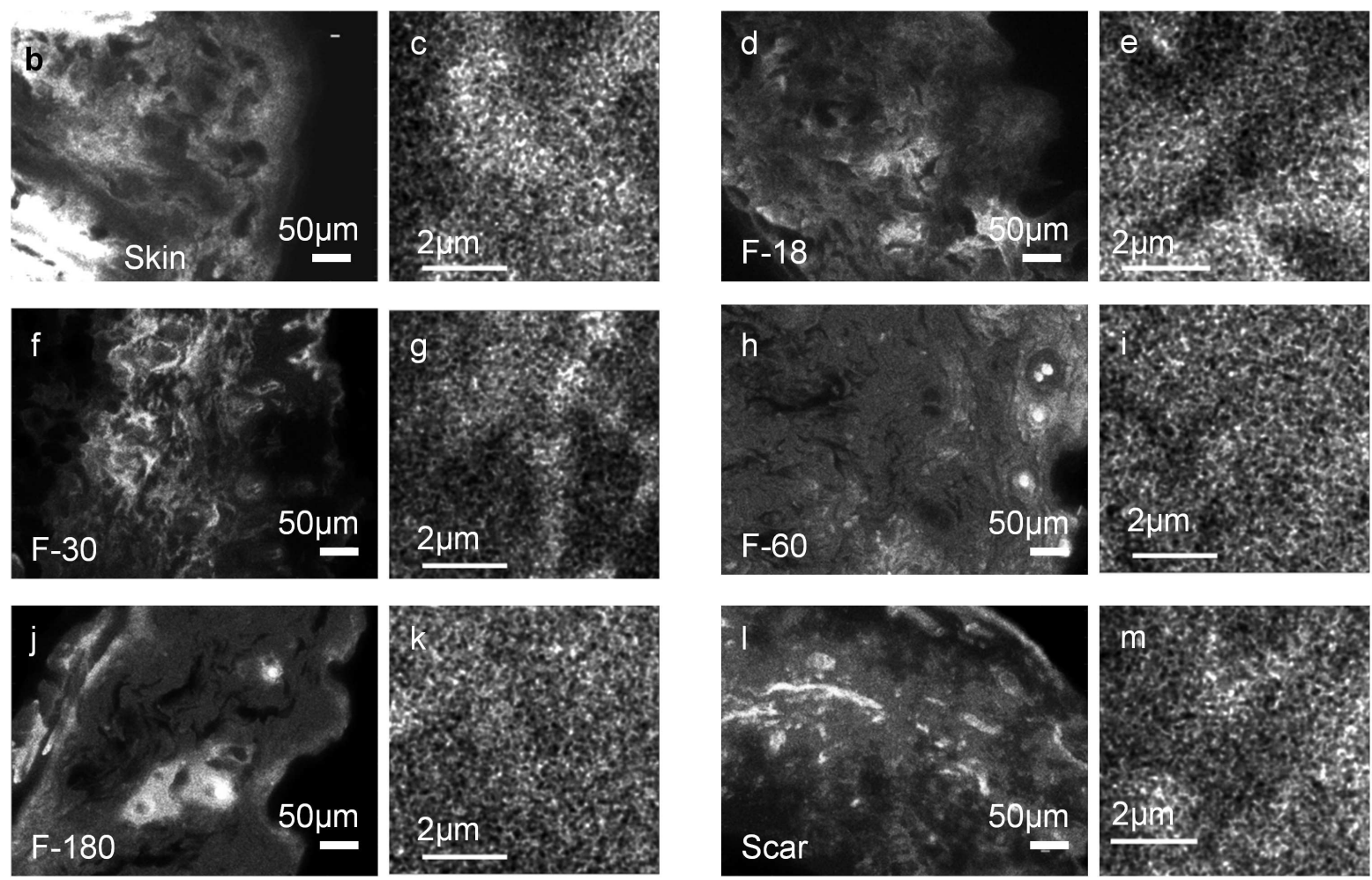

Figure 7: tAF-MUSICAL for wound healing scars and pathological fibrosis. (a) a pictorial representation of mice skin and changes it undergoes during arecanut-induced pathological fibrosis and scar fibrosis. A broad and small field of tAF-MUSICAL from the same sample for $(b, c)$ healthy skin. tAF-MUSICAL of pathological fibrosis by arecanut extract (ANE) after application for (d,e) 18 days, (f,g) 30 days, (h,i) 60 days, and (j,k) 180 days. (l,m) broad and small tAF-MUSICAL field views of a scar after 60 days of a full-thickness skin injury. All experiments performed on dorsal mice skin. 

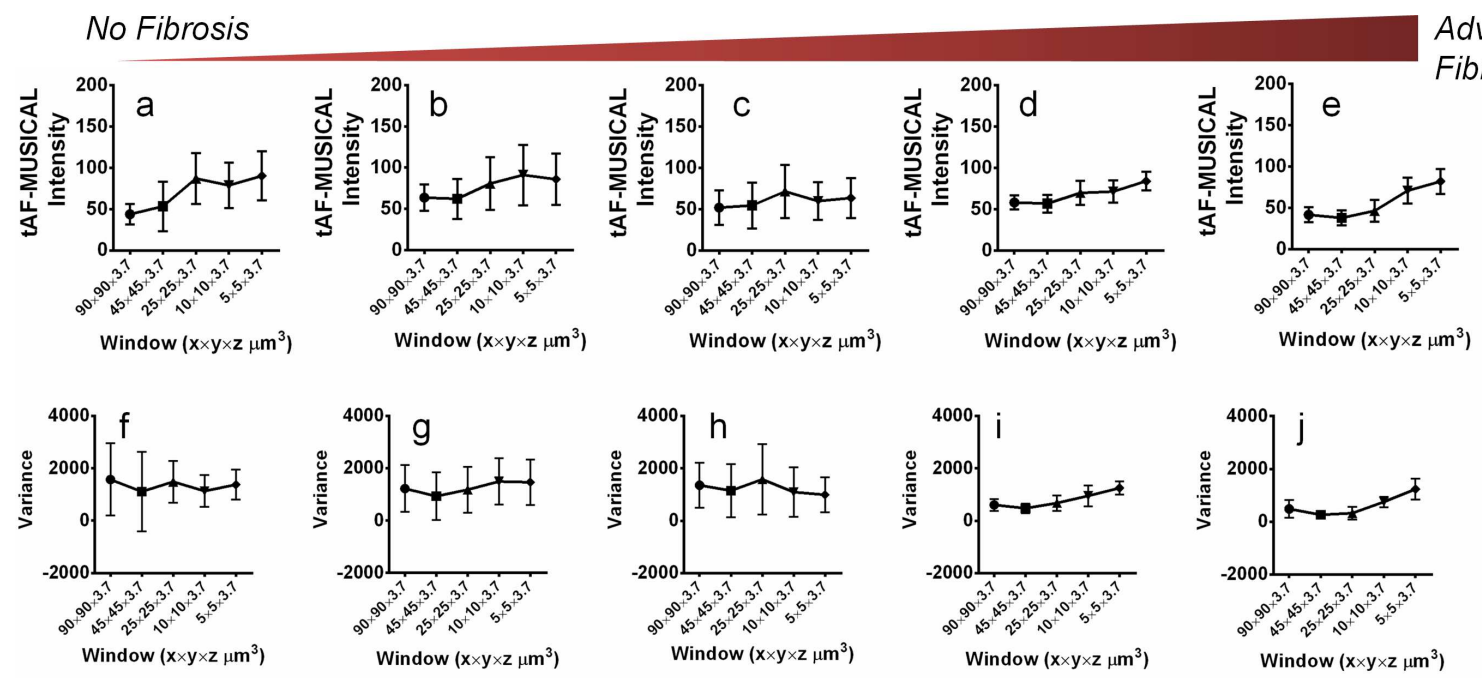

Large Field
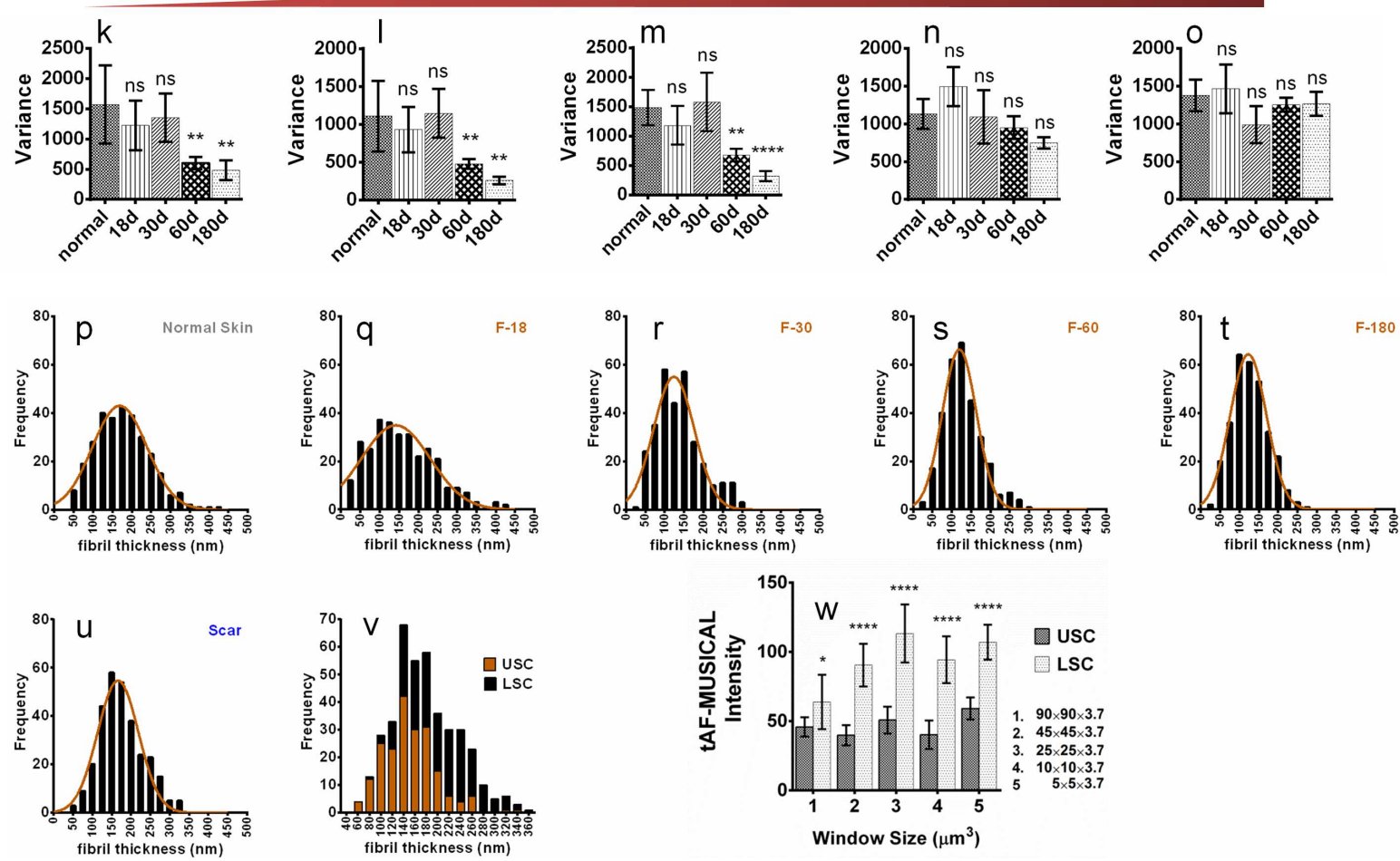

Small Field

Figure 8: Multi-scale pathological evaluation of fibrosis with tAF-MUSICALtAFMUSICAL measurement of (a-e) intensities and (f-j) variance different window sizes standard deviation resembling variations within and between the windows; $(\mathrm{a}, \mathrm{f})$ healthy skin, $(\mathrm{b}, \mathrm{g}) 18$ day fibrosis, (c,h) 30-day fibrosis (d,i) 60-day fibrosis, and (e,j) 180-day fibrosis. (k-o) shows variance measurements between fibrotic stages, with window sizes (k) $90 \times 90 \times 3.7 \mu \mathrm{m}^{3}$, (l) $45 \times 45 \times 3.7 \mu m^{3}$, (m) $25 \times 25 \times 3.7 \mu m^{3}$, (n) $10 \times 10 \times 3.7 \mu m^{3}$, (o) $5 \times 5 \times 3.7 \mu m^{3}$. (pu) tAF-MUSICAL derived histograms of fibrosis stages in skin tissues and scar, (v) histogram of two scar compartments, (w) tAF-MUSICAL intensity differences in a pair of scar compartments from same tissue collected from different window sizes. $\mathrm{N}=30$ per group, All plots (k-o, w) are represented as $\mu \pm \mathrm{SD}$, ns (not significant) $\mathrm{p}>0.05,{ }^{*} \mathrm{p} \leq 0.05, * * \mathrm{p} \leq 0.01, * * * \mathrm{p} \leq 0.001$, $* * * * \mathrm{p} \leq 0.0001$. For statistical analysis we used one-way ANOVA (95\% confidence interval) followed by Tukey's post hoc test. 


\section{Figures}
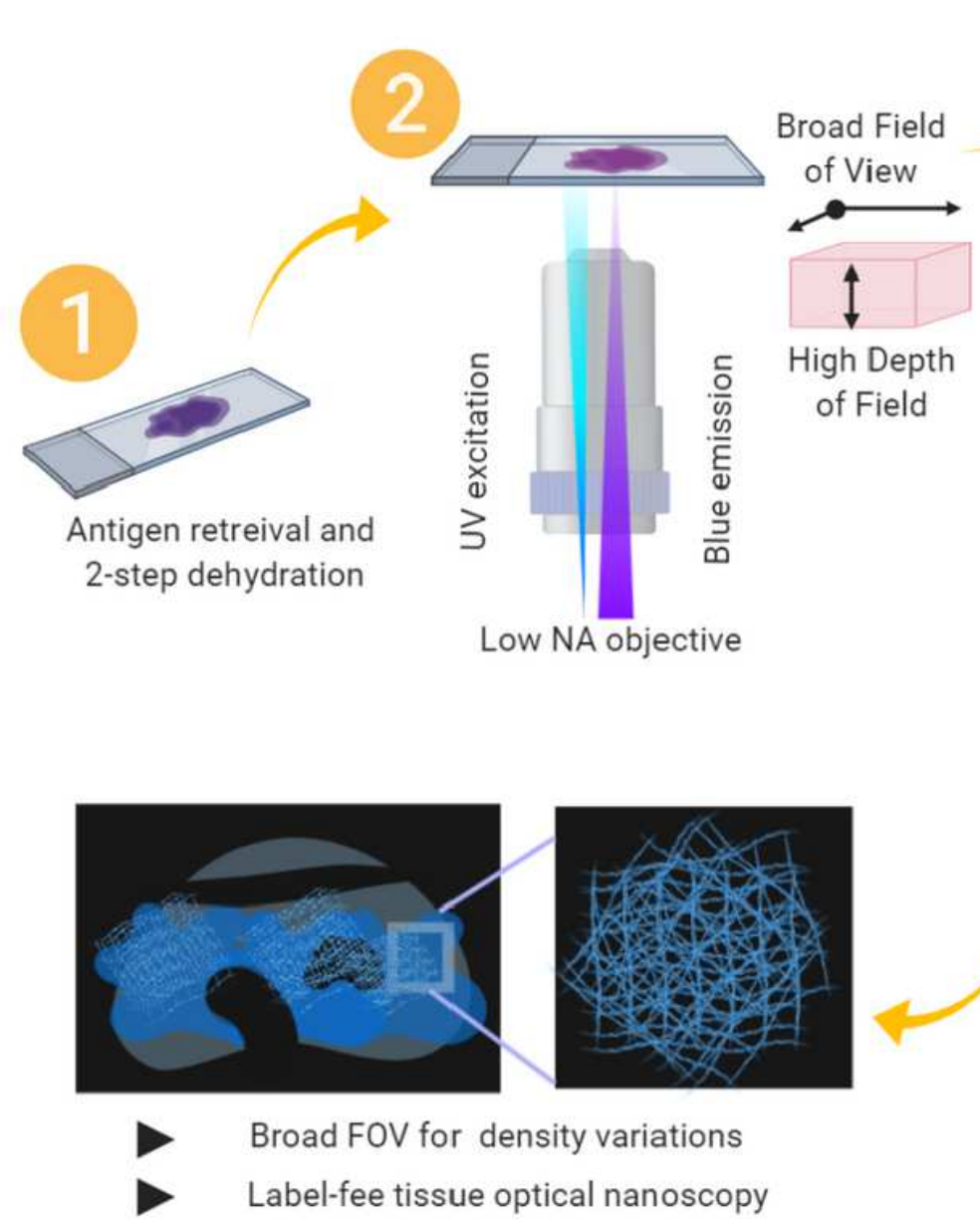

Broad FOV for density variations
Label-fee tissue optical nanoscopy
tAF fluctuations as spatial intensity variations on temporally spaced images

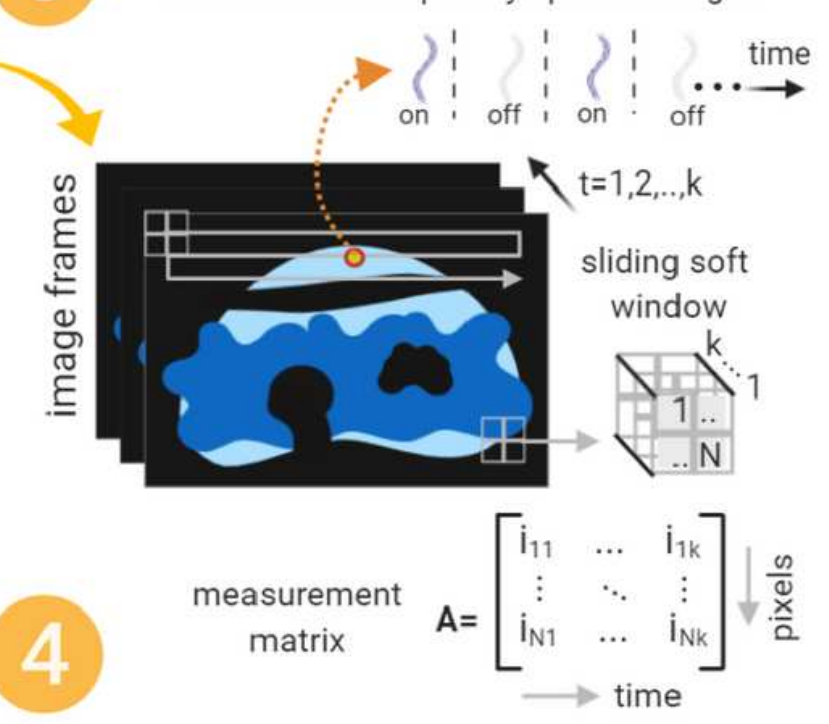

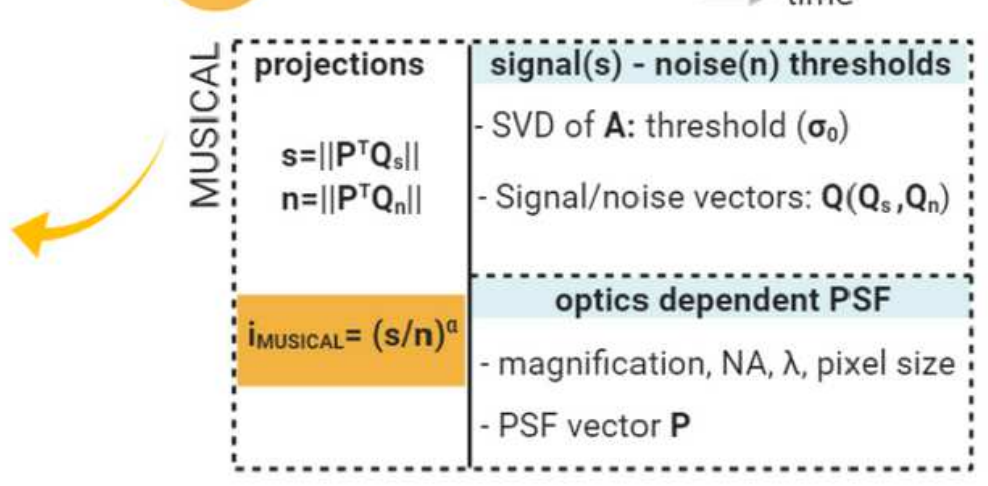

\section{Figure 1}

Schematic diagram for tAF-MUSICAL workflow The FFPE tissue sections replete with autofluorescing matrix proteins are mounted on glass slides, deparaffinised and completely dehydrated. Antigen retrieval is performed to remove unwanted fixative-linked fluorescence. A low NA epifluorescence microscope (20 $\bigotimes, 0.40$ NA objective) that allows a broad field of view (FOV) of $\sim 323 \times 435 \mu \mathrm{m} 2$ and a thicker depth of field (DOF) of $\sim 3.7 \mu \mathrm{m}$ is used to acquire image stacks at excitation and emission wavelengths specific to matrix protein autofluorescence. The rapidly acquired image stacks are processed by MUSICAL to achieve super-resolution as shown and previously described (19). The tAF-MUSICAL image presents density variations on a broad field due to accumulated autofluorescence of high DOF. The tAF-MUSICAL image can be zoomed in for visualizing fibril nano-structures with dimensions as low as $\sim 43 \mathrm{~nm}$. 

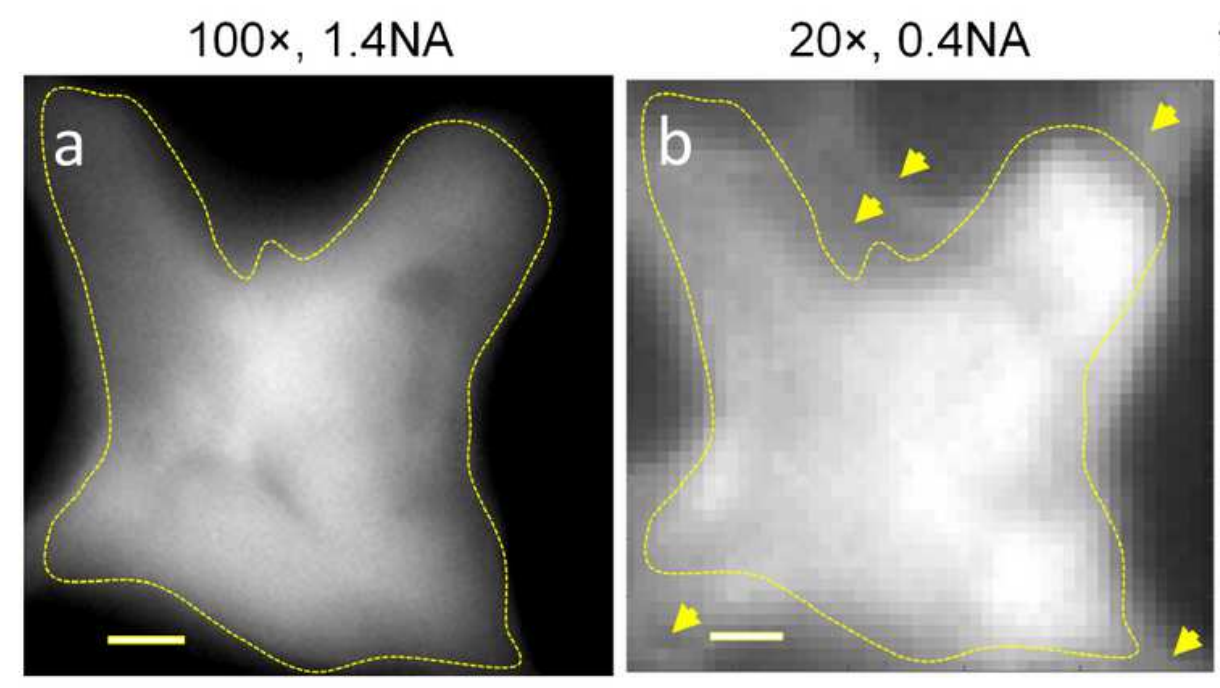

tAF-MUSICAL $(20 \times, 0.4 N A)$
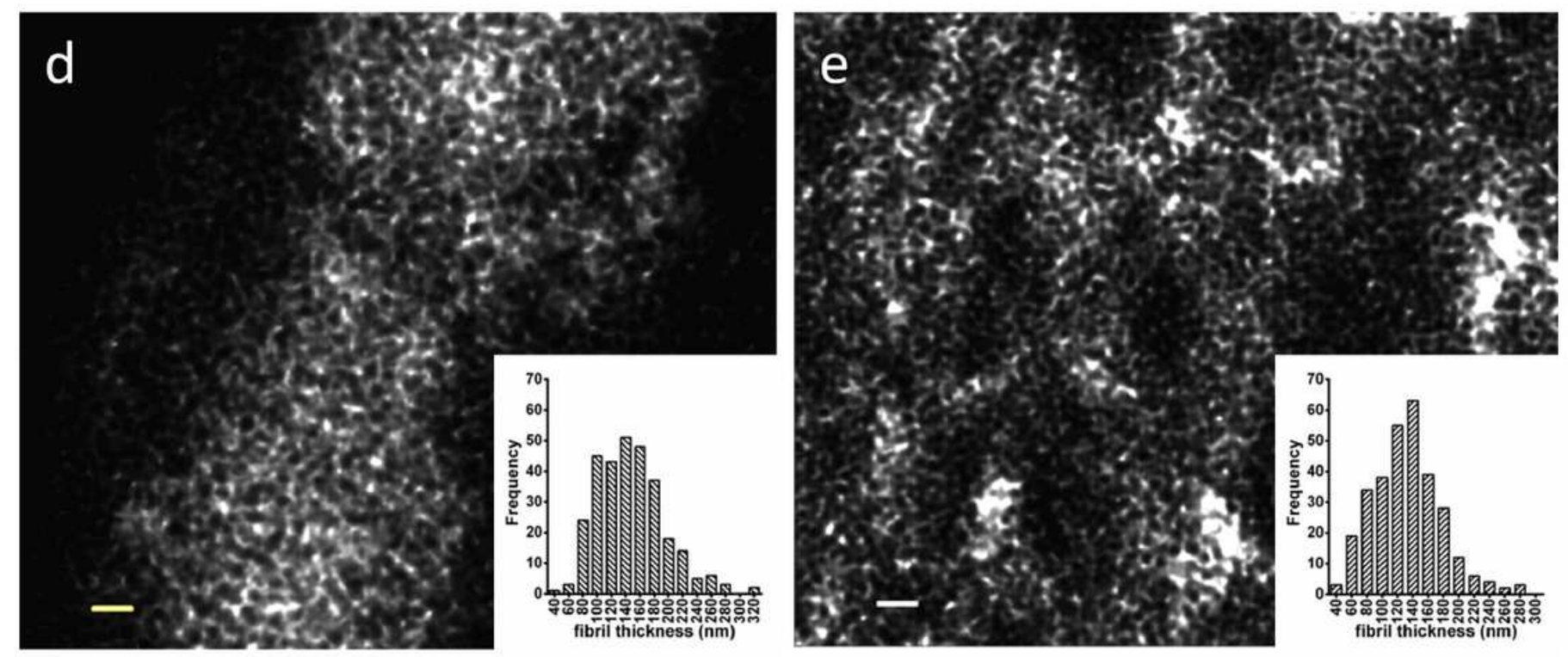

Figure 2

Comparing tAF-MUSICAL super-resolution with diffraction-limited tissue autofluorescence imaging. A region from skin tissue imaged with (a) a $1.40 \mathrm{NA}$ (oil) $100 \mathrm{x}$ objective and (b) a $0.40 \mathrm{NA}, 20 \mathrm{x}$ objective on an epifluorescence microscope. (c) tAF-MUSICAL super-resolution obtained from image frames collected by a 20x, 0.4 NA epifluorescence microscope objective; yellow arrows show exclusive regions in low NA objective, yellow dotted line is the high NA object's region boundary (d) tAF-MUSICAL image region showing keratin rich-epidermal layer of mouse skin tissue. (e) tAF-MUSICAL image region showing collagen rich-dermis of mouse skin tissue. In (d) and (e), inset shows the distribution of respective fibril diameters. scale bar $=2 \mu \mathrm{m}$. 

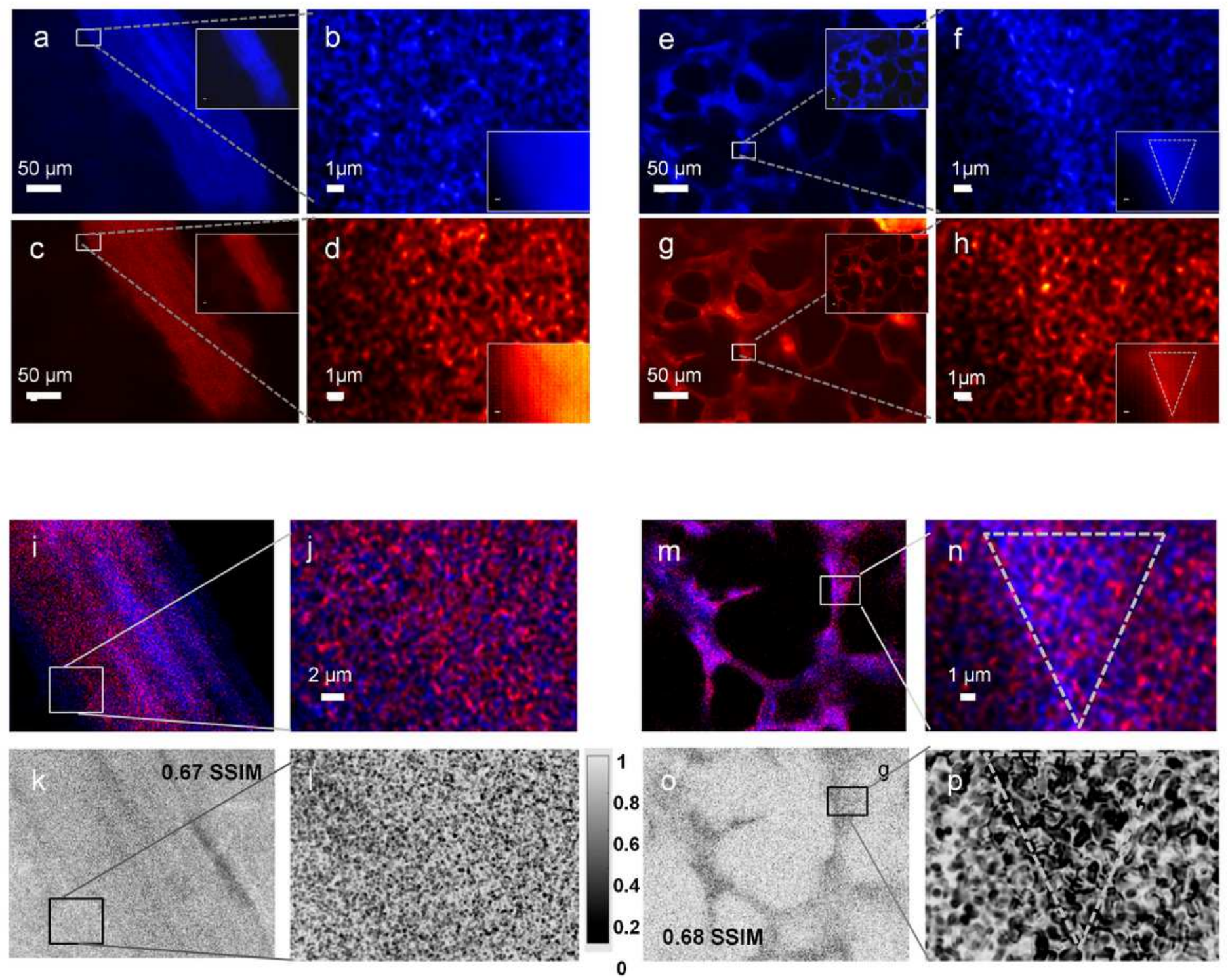

Figure 3

Molecular specificity of tAF-MUSICAL in detecting collagen. MUSICAL superresolution images of (a-d) purified collagen-I and (e-h) skin tissue section imaging. Images derived from (a,b,e,f) tissue autofluorescence (tAF) in blue emission, (c,d,g,h) same tissue immunolabeled with collagen-I and fluorescent probe having red emission. All insets show the corresponding source (diffraction-limited) images (20x, 0.4 NA objective). (a,c,e,g) are full-field MUSICAL images and (b,d,f,h) are a small region illustrating super-resolved structures. Colocalization of MUSICAL structures of $(\mathrm{i}, \mathrm{j})$ pure Collagen-I and $(m, n)$ tissue from auto (blue) and labeled (red) fluorescence. Similarity maps of MUSICAL structures in the red and blue colors images in $(\mathrm{i}, \mathrm{j})$ are shown in $(\mathrm{k}, \mathrm{l})$ for Collagen-l, and similarly similarity maps of the structures in red and blue colored images in $(m, n)$ are shown in $(o, p)$ for the skin tissue. 


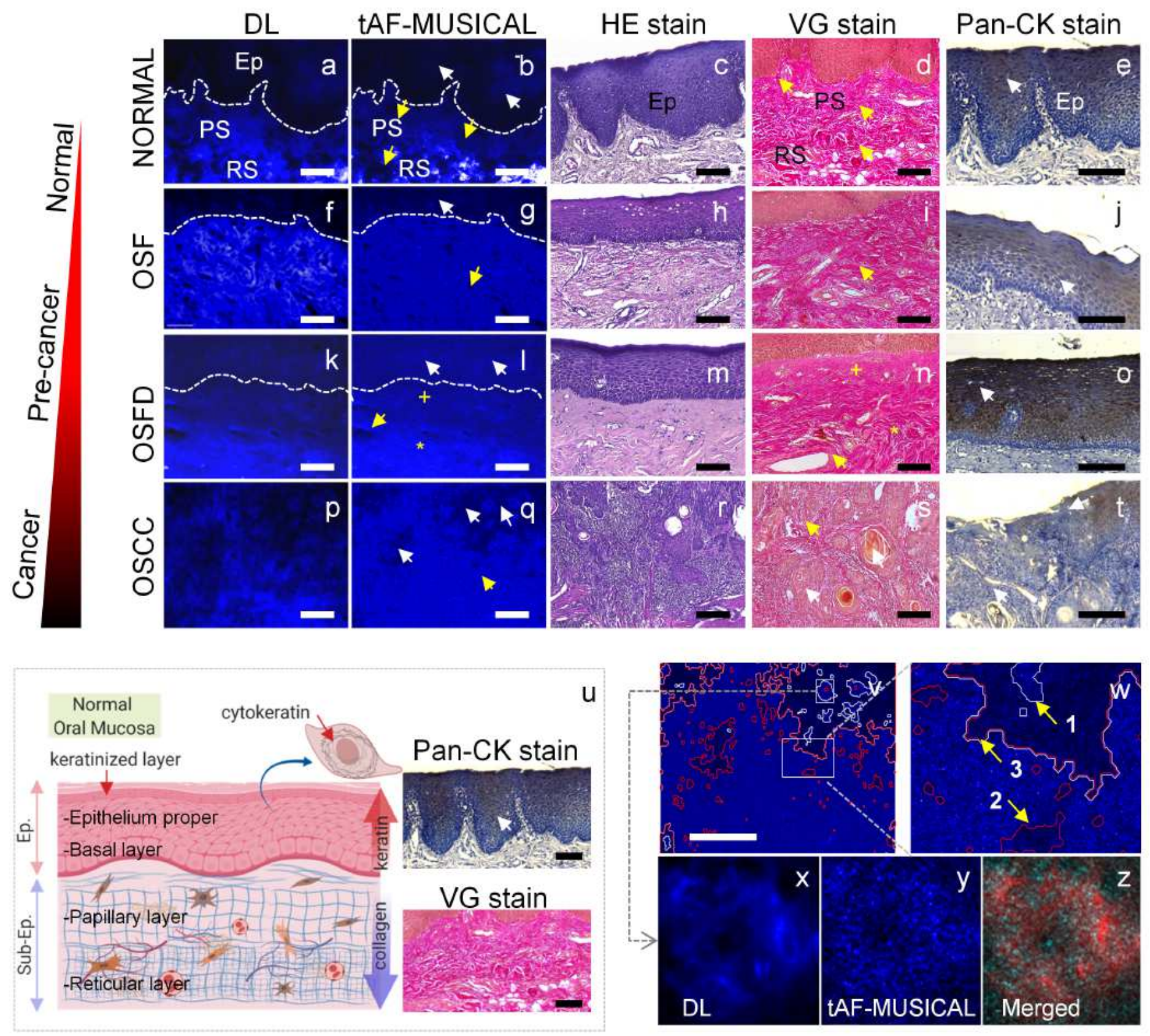

Figure 4

tAF-MUSICAL in imaging pre-cancer progression in the oral mucosa. Corresponding diffraction-limited (DL) image, tAF-MUSICAL, Hematoxylin and Eosin (HE) stained (gold standard), van Gieson (VG) stained (for all collagen in sub-epithelium), and pan-keratin (for all keratin in epithelium) stained sections of (a-e) normal oral mucosa (NOM), ( $f-j$ ) oral submucous fibrosis (OSF), (k-o) OSF with dysplasia (OSFD), (p-t) oral squamous cell carcinoma (OSCC). The yellow arrows and markers indicate sub-epithelial changes in tAF-MUSICAL corroborative with ground truth images; the white arrows mark groud truth matching epithelial changes. ( $u$ ) the anatomical structure of oral mucosa includes keratin and collagen's localization (with stained images for reference) in different layers of epithelium and sub-epithelium. (v) tAF-MUSICAL of OSCC with the invasions of epithelial tissues in sub-epithelium,(w) an ROI of OSCC showing: epithelial islands in the subepithelium (red line), keratin pearls within the epithelium (white line) 
and the non-disrupted basement membrane (red-epithelial side, white -sub-epithelial side). ( $x, y, z)$ The DL, tAF-MUSICAL, and merged image of a keratin pearl (a mark of carcinoma). Ep = epithelium, Sub-Ep = sub-epithelium, PS=papillary sub-epithelium, RS=reticular sub-epithelium. scale bars $=50 \mu \mathrm{m}$

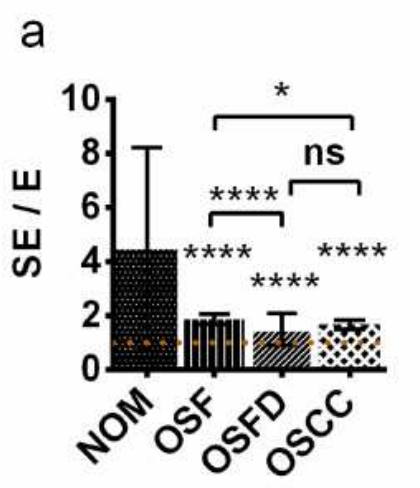

b

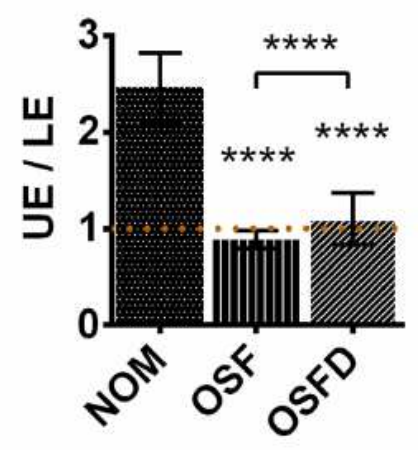

C

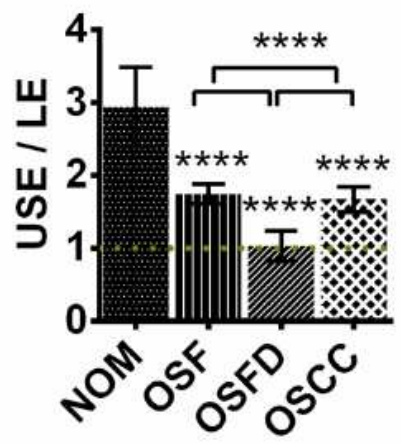

d

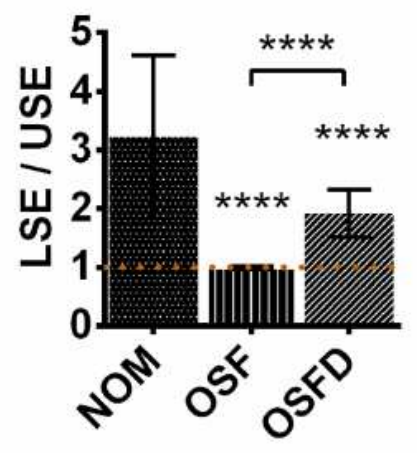

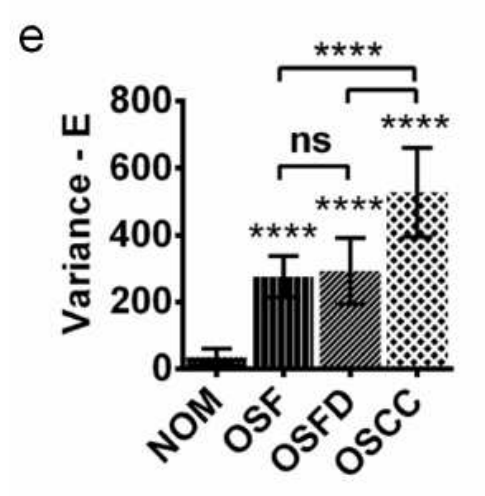

h

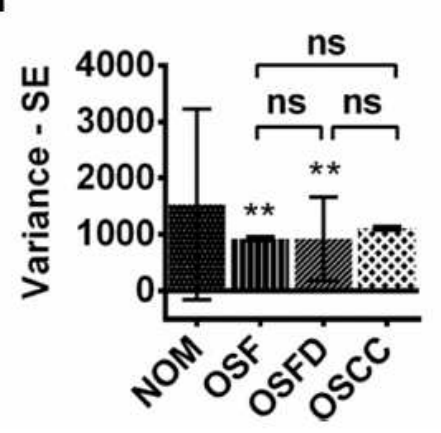

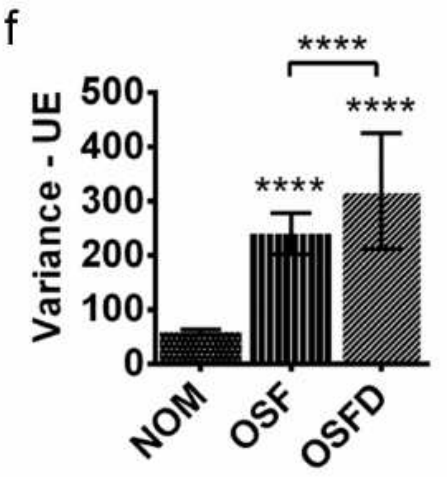

i

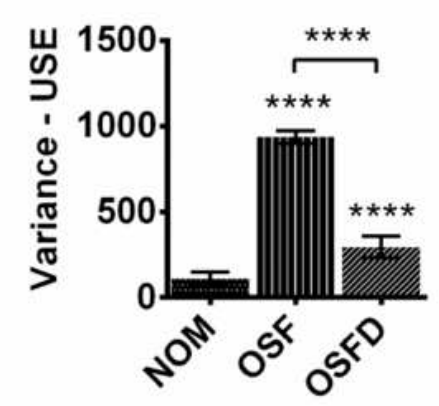

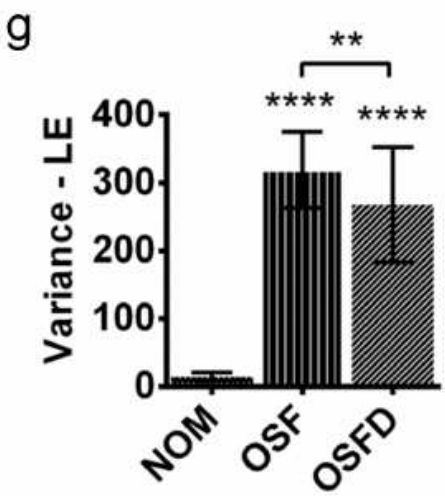

j

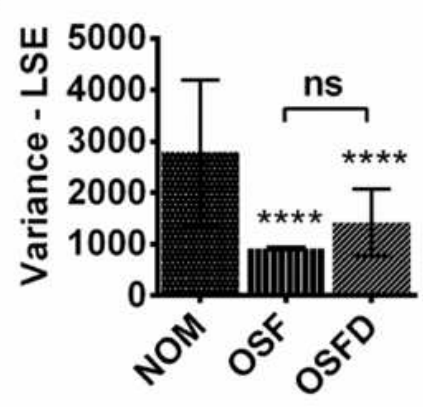

Figure 5

Quantification of broad-field tAF-MUSICAL features in delineating oral precancer stages. (a-d) ratio of intensity between the layers and sub-layers of epithelium and sub-epithelium to delineate the stagesNOM, OSF, OSFD, and OSCC. (e-j) variance quantification of different layers and sub-layers of epithelium and sub-epithelium to delineate the oral pre-cancer stages. In OSCC tissues, the epithelium and subepithelium cannot be differentiated due to metastasis and hence were not included in $(b, d, f, g, i, j)$. Eepithelium whole, UE-upper epithelium, LE-lower epithelium, SE-sub-epithelium whole, USE-upper subepithelium, LSE-lower sub-epithelium. UE, LE, USE, LSE of tAF-MUSICAL histologically represent 
epithelium proper, basal/supra-basal epithelium, papillary sub-epithelium and reticular sub-epithelium respectively. $\mathrm{N}=30$ per group, All plots are represented as $\mu \pm S D$, ns (not significant) $p>0.05, * p \leq 0.05$, ** $p \leq 0.01,{ }^{* \star *} p \leq 0.001,{ }^{\star \star \star \star} p \leq 0.0001$. For statistical analysis we used one-way ANOVA ( $95 \%$ confidence interval) followed by Tukey's post hoc test.

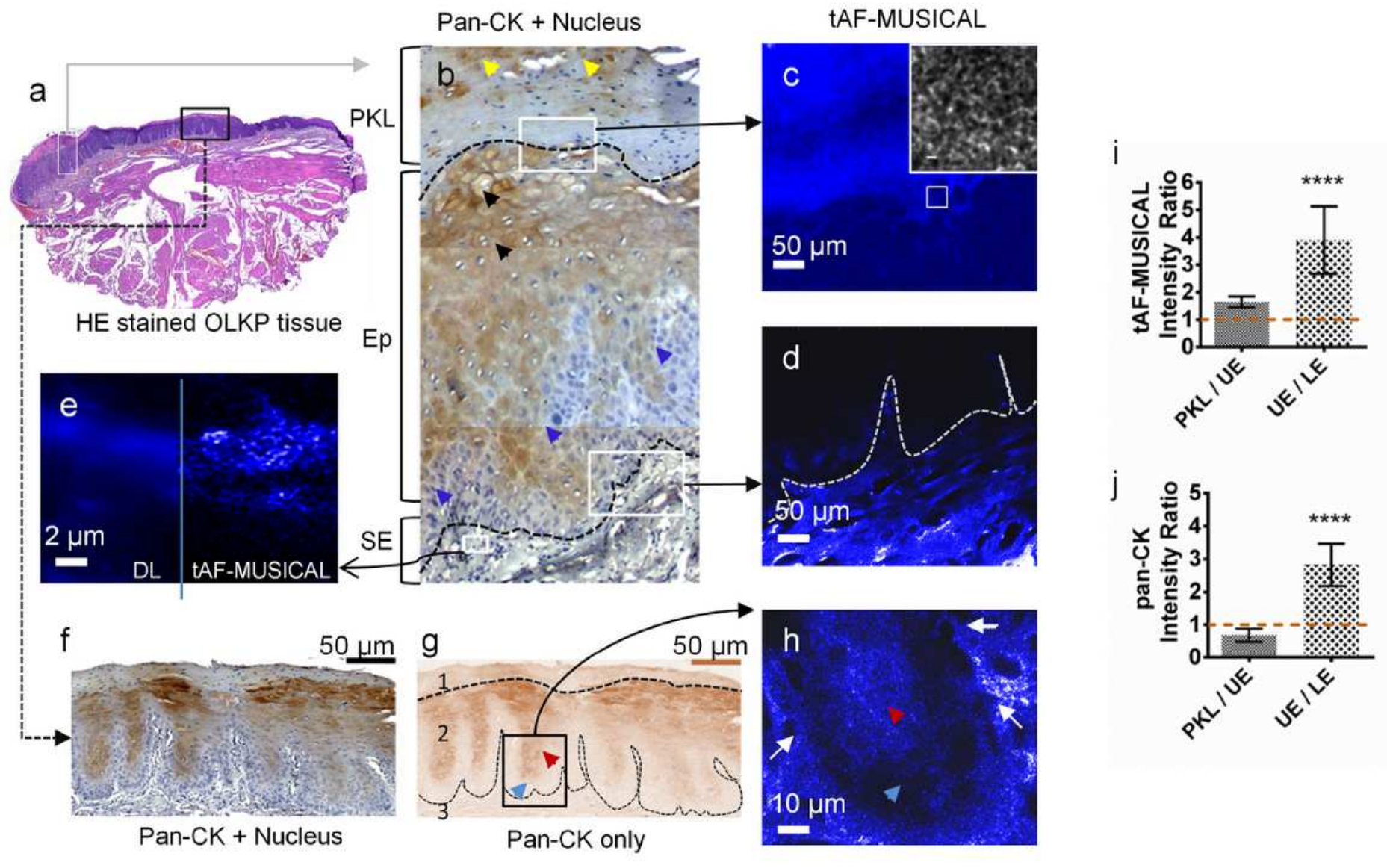

Figure 6

tAF-MUSICAL in detecting keratin associated oral leukoplakia. MUSICAL super-resolution images of (a-d) purified collagen-l and (e-h) skin tissue section imaging. Images derived from $(a, b, e, f)$ tissue autofluorescence (tAF) in blue emission, (c,d,g,h) same tissue immunolabeled with collagen-I and fluorescent probe having red emission. All insets show the corresponding source (diffraction-limited) images (20x, 0.4 NA objective). (a,c,e,g) are full-field MUSICAL images and (b,d,f,h) are a small region illustrating super-resolved structures. $N=30$ per group, All plots are represented as $\mu \pm S D$, $\star \star \star \star ~ p \leq 0.0001$. For statistical analysis we used two-tailed t-test (95\% confidence interval). 

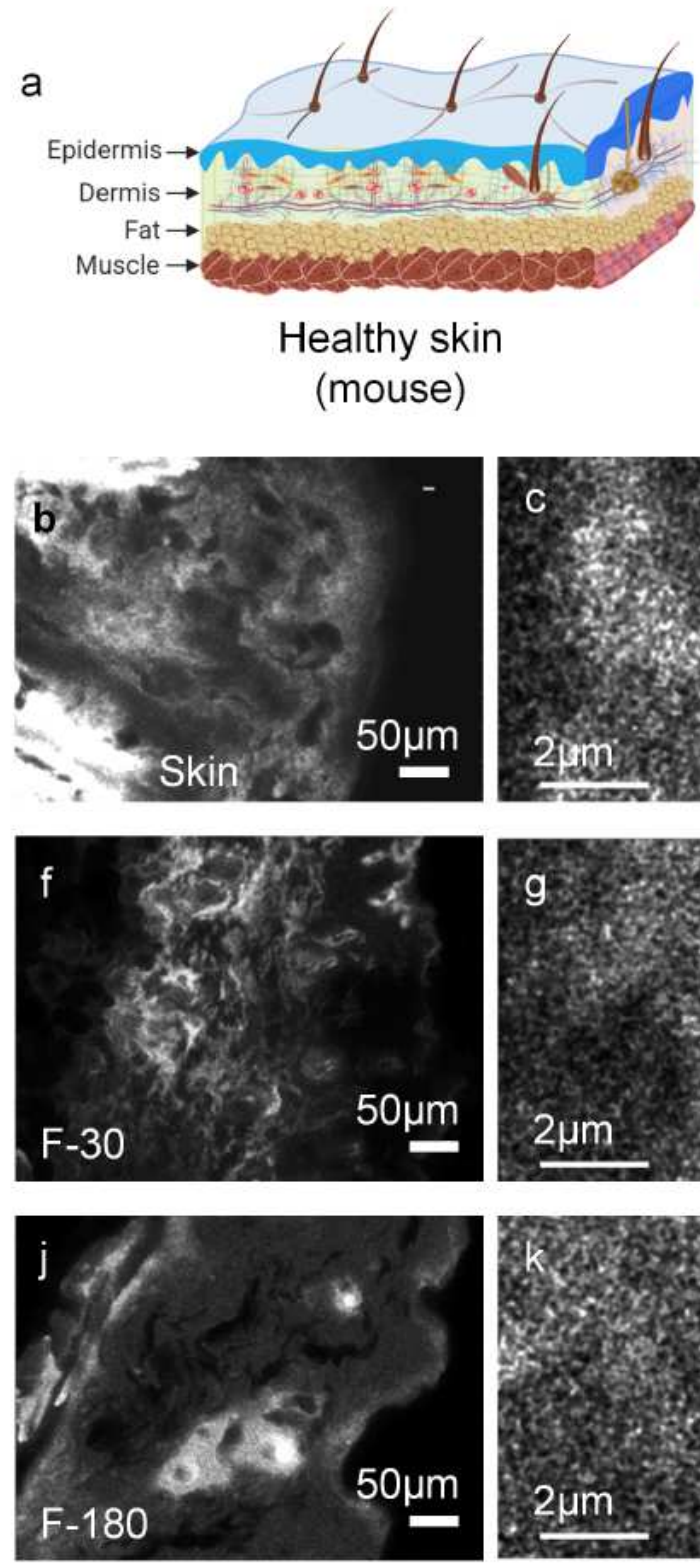

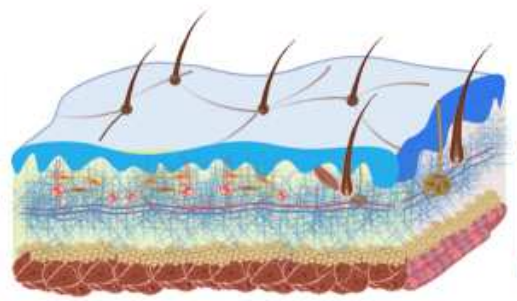

Arecanut-induced (pathological) fibrosis

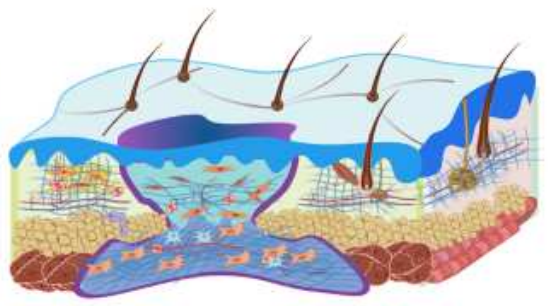

Post-wound scar tissue
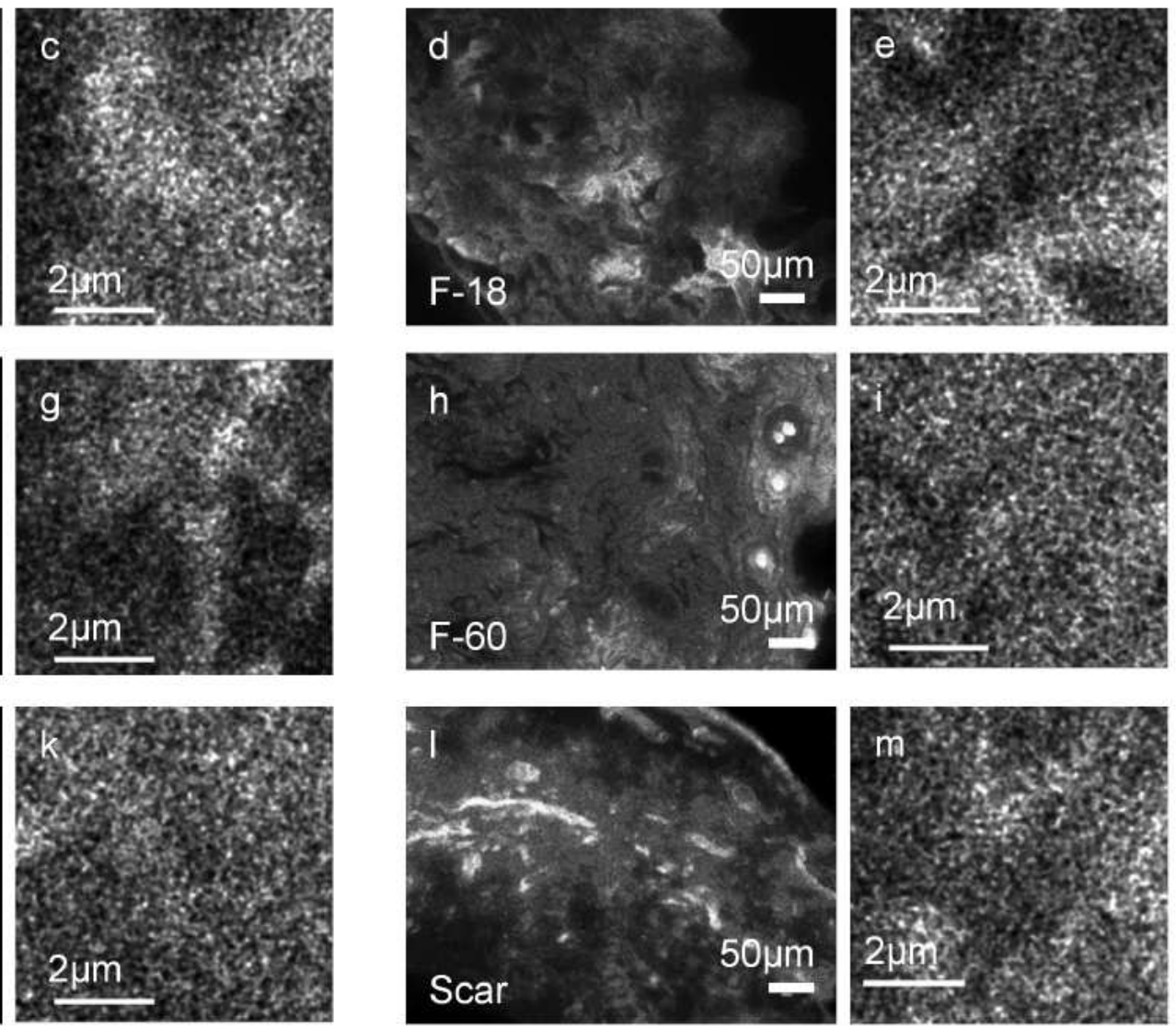

\section{Figure 7}

tAF-MUSICAL for wound healing scars and pathological fibrosis. (a) a pictorial representation of mice skin and changes it undergoes during arecanut-induced pathological fibrosis and scar fibrosis. A broad and small field of tAF-MUSICAL from the same sample for $(b, c)$ healthy skin. TAF-MUSICAL of pathological fibrosis by arecanut extract (ANE) after application for (d,e) 18 days, $(f, g) 30$ days, $(h, i) 60$ days, and (j,k) 180 days. (l,m) broad and small tAF-MUSICAL field views of a scar after 60 days of a fullthickness skin injury. All experiments performed on dorsal mice skin. 

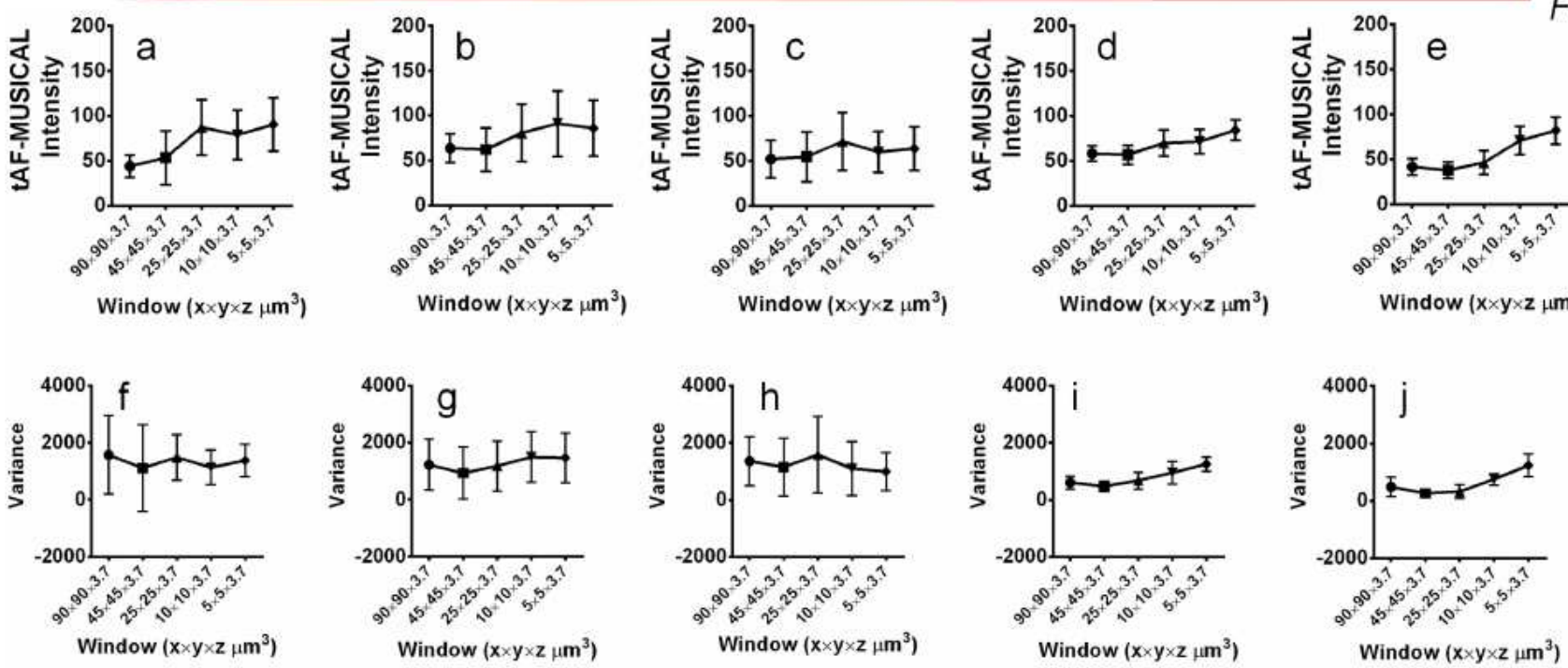

Large Field
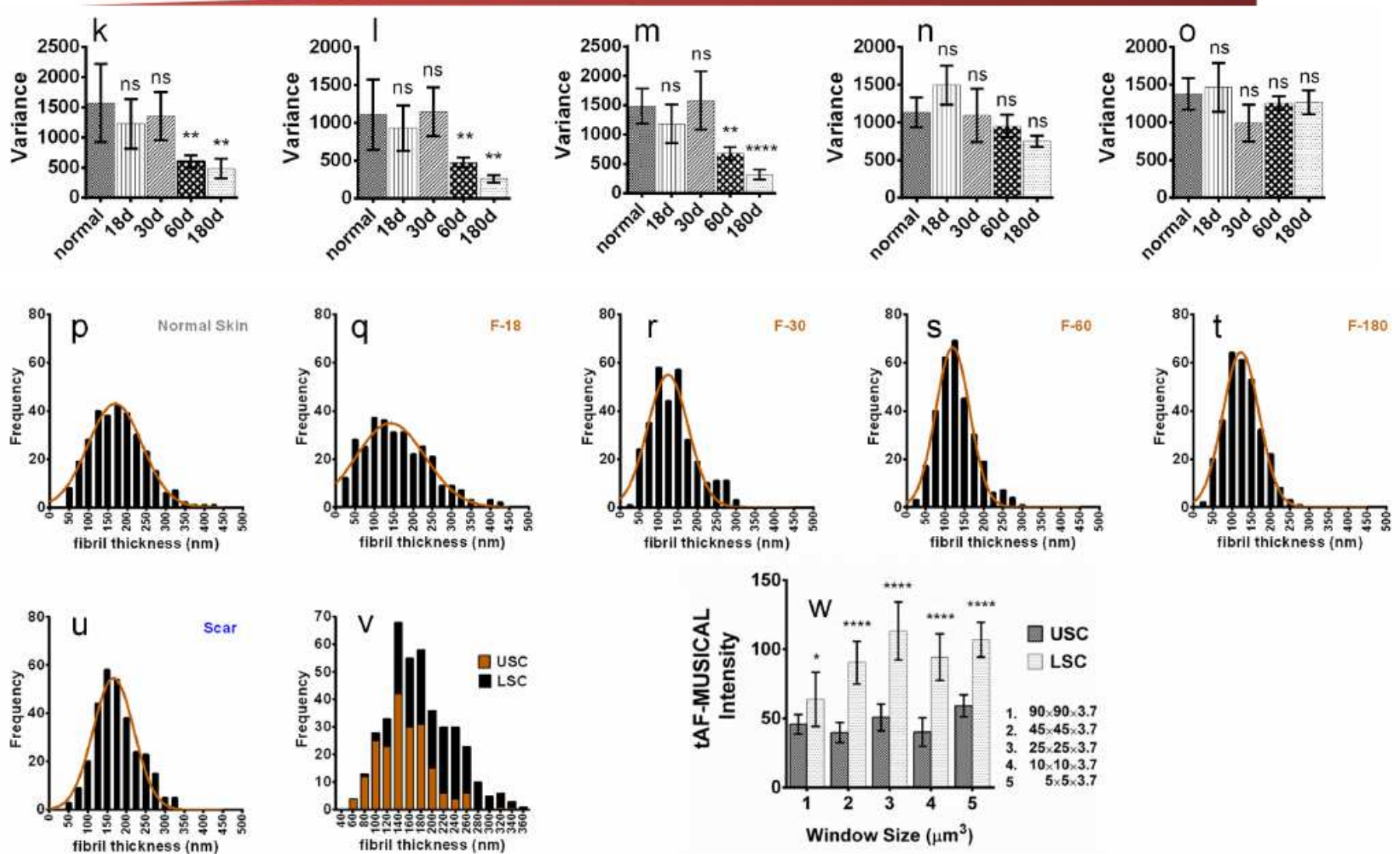

Small Field 
3.7 $\mu \mathrm{m3}$. (pu) tAF-MUSICAL derived histograms of fibrosis stages in skin tissues and scar, (v) histogram of two scar compartments, (w) tAF-MUSICAL intensity differences in a pair of scar compartments from same tissue collected from different window sizes. $N=30$ per group, All plots $(k-0, w)$ are represented as $\mu$ $\pm S D$, ns (not significant) $p>0.05, * p \leq 0.05, * * p \leq 0.01, * \star * p \leq 0.001, * \star \star * p \leq 0.0001$. For statistical analysis we used one-way ANOVA ( $95 \%$ confidence interval) followed by Tukey's post hoc test.

\section{Supplementary Files}

This is a list of supplementary files associated with this preprint. Click to download.

- tAFMUSICALSupplementary.pdf 